for

\title{
Palladium-Catalyzed Regio- and Stereoselective Carbothiolation of Terminal Alkynes with Azolyl Sulfides
}

\author{
Masayuki Iwasaki, ${ }^{\dagger}$ Nikola Topolovčan, ${ }^{\dagger}$ Hao Hu,${ }^{\dagger}$ Yugo Nishimura, ${ }^{\dagger}$ \\ Glwadys Gagnot, ${ }^{\dagger}$ Rungsaeng Na nakorn, ${ }^{\dagger}$ Ramida Yuvacharaskul, ${ }^{\dagger}$ \\ Kiyohiko Nakajima ${ }^{\ddagger}$, and Yasushi Nishihara* ${ }^{\dagger}$ \\ ${ }^{\dagger}$ Division of Earth, Life, and Molecular Science, \\ Graduate School of Natural Science and Technology, \\ Okayama University, 3-1-1 Tsushimanaka, Kita-ku, Okayama 700-8530, Japan \\ ${ }^{*}$ Department of Chemistry, Aichi University of Education, \\ Igaya, Kariya 448-8542, Japan \\ Phone: $+81-86-251-7855$ \\ Fax: +81-86-251-7855 \\ Email: ynishiha@okayama-u.ac.jp
}

Contents

1. General S2

2. Optimization of Reaction Conditions S3

3. Experimental Procedures and Spectroscopic Data for New Compounds S4-S13

4. Copies of ${ }^{1} H$ and ${ }^{13} C$ NMR Charts for the New Compounds S14-S40

5. A Plausible Reaction Mechanism of Allylation of Sulfoxide $6 \quad S 41$

6. Structural Determination of Compounds by X-ray Analysis S42-S45

7. References $\$ 46$ 


\section{General}

\section{Instrumentation}

All the reactions were carried out under an Ar atmosphere using standard Schlenk techniques. Glassware was dried in an oven $\left(150^{\circ} \mathrm{C}\right)$ and heated under reduced pressure before use. For thin layer chromatography (TLC) analyses throughout this work, Merck precoated TLC plates (silica gel $60 \mathrm{~F}_{254}, 0.25 \mathrm{~mm}$ ) were used. Silica gel column chromatography was carried out using Silica gel 60 (spherical, 40-100 $\mu$ m) from Kanto Chemicals Co., Ltd. NMR spectra $\left({ }^{1} \mathrm{H},{ }^{13} \mathrm{C}\left\{{ }^{1} \mathrm{H}\right\}\right.$, and $\left.{ }^{19} \mathrm{~F}\left\{{ }^{1} \mathrm{H}\right\}\right)$ were recorded on Varian INOVA-600 (600 MHz), Mercury-400 (400 MHz) and 300-NMR ASW $(300 \mathrm{MHz})$ spectrometers. Chemical shifts $(\delta)$ are in parts per million relative to $\mathrm{CDCl}_{3}$ at 7.26 ppm for ${ }^{1} \mathrm{H}$ and at $77.0 \mathrm{ppm}$ for ${ }^{13} \mathrm{C}\left\{{ }^{1} \mathrm{H}\right\}$ NMR spectra or DMSO- $d_{6}$ at $2.50 \mathrm{ppm}$ for ${ }^{1} \mathrm{H}$ and at 39.5 ppm for ${ }^{13} \mathrm{C}\left\{{ }^{1} \mathrm{H}\right\}$ NMR spectra, respectively. The ${ }^{19} \mathrm{~F}\left\{{ }^{1} \mathrm{H}\right\}$ NMR spectra were measured by using $\mathrm{C}_{6} \mathrm{H}_{5} \mathrm{~F}(\delta=-113.15)$ as an external standard. The NMR yields were determined using dibromomethane as internal standard. Infrared spectra were recorded on a Shimadzu IRPrestige-21 spectrophotometer and reported in wave numbers $\left(\mathrm{cm}^{-1}\right)$. LRMS analyses were carried out on a SHIMADZU GC-17A equipped with a SHIMADZU QP-5050 GC-MS system. HRMS were determined on a JEOL JMS-700 MStation. Elemental analyses were carried out with a Perkin-Elmer $2400 \mathrm{CHN}$ elemental analyzer.

The reaction under microwave irradiation was carried out using a focused microwave unit (Biotage Initiator $^{\mathrm{TM}}$ ). The maximum irradiation power is $400 \mathrm{~W}$. Each reaction was run in a 20-mL glass pressure vial, which is a commercially available vial specifically for the Biotage Initiator ${ }^{\mathrm{TM}}$. It took $5 \mathrm{~min}$ to reach $160{ }^{\circ} \mathrm{C}$. After reaching the indicated temperature, controlled microwave irradiation started and continued for $40 \mathrm{~min}$, keeping the reaction temperature constant.

\section{Chemicals}

Unless otherwise noted, materials obtained from commercial suppliers were used without further purification. Pd-PEPPSIs ${ }^{1}$ was prepared according to the literature. Sulfides $\mathbf{1 a}$ and $\mathbf{1 d}-\mathbf{1} \mathbf{g}$ were prepared by the reaction of the corresponding thiol with iodomethane. ${ }^{2}$ Sulfide $\mathbf{1 b}$ was prepared from benzothiazole and diphenyl disulfide. ${ }^{3} \quad$ Sulfides $\mathbf{1 a},{ }^{4} \mathbf{1 b},{ }^{5} \mathbf{1 d},{ }^{6} \mathbf{1 e},{ }^{7} \mathbf{1 f},{ }^{8}$ and $\mathbf{1} \mathbf{g}^{9}$ were known compounds. Sulfide $\mathbf{1 c}$ and alkynes $\mathbf{2}$ were commercially available. 


\section{Optimization of Reaction Conditions}

\section{Additive Screening}

2a (2 equiv)
entry




\section{Experimental Procedures and Spectroscopic Data for New Compounds}

A Typical Procedure for Addition of Azolyl Sulfides 1 to Terminal Alkynes 2: Synthesis of (Z)-2-(2-Methylthio-2-phenylethenyl)benzothiazole (3aa).
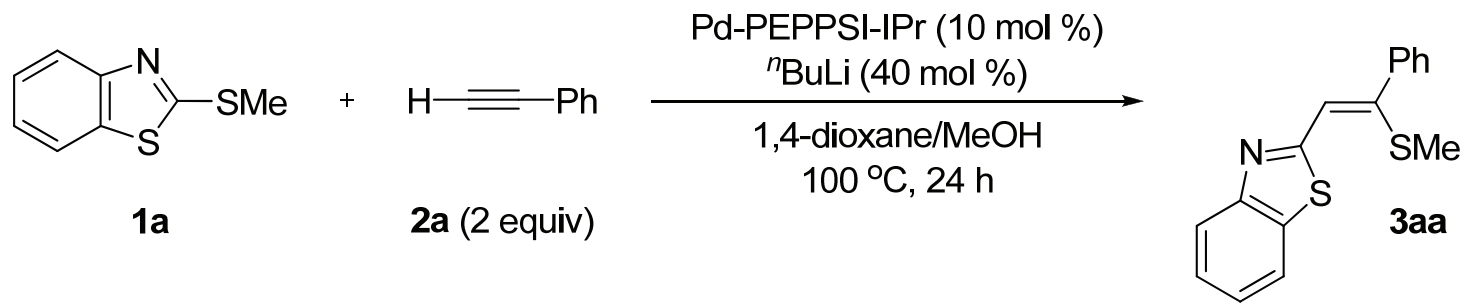

Pd-PEPPSI-IPr (68 mg, $0.10 \mathrm{mmol}$ ) was placed in a 20-mL Schlenk tube under argon. $n$-Butyllithium (1.6 M hexane solution, $0.25 \mathrm{~mL}, 0.40 \mathrm{mmol})$ and 1,4-dioxane $(8 \mathrm{~mL})$ were added, and the mixture was stirred for $15 \mathrm{~min}$. Phenylacetylene (2a, $220 \mu \mathrm{L}, 2.0 \mathrm{mmol})$, 2-benzothiazolyl methyl sulfide $(1 \mathrm{a}, 181 \mathrm{mg}, 1.0 \mathrm{mmol})$, and methanol $(250 \mu \mathrm{L})$ were sequentially added. The reaction mixture was heated at $100{ }^{\circ} \mathrm{C}$ for $24 \mathrm{~h}$. After the mixture was cooled to $25^{\circ} \mathrm{C}$, the reaction was quenched with saturated $\mathrm{NH}_{4} \mathrm{Cl}$ aqueous solution $(10 \mathrm{~mL})$ and extracted with ethyl acetate $(10$ $\mathrm{mL} \times 3$ ). The combined organic layers were dried over anhydrous sodium sulfate and concentrated under reduced pressure. Purification of the residue on silica gel column chromatography (hexane:ethyl acetate $=20: 1)$ provided 3aa $(263 \mathrm{mg}, 0.928 \mathrm{mmol})$ in $93 \%$ yield as a yellow viscous oil.

$\mathrm{R}_{f}=0.21$ (hexane:ethyl acetate $=20: 1$ ). $\quad$ B.p. $150^{\circ} \mathrm{C} / 9.8$ Torr. FT-IR (neat, $\mathrm{cm}^{-1}$ ): $3055(\mathrm{w}), 2920$ (w), 2849 (w), 1427 (m), 1317 (w), 758 (s). ${ }^{1} \mathrm{H}$ NMR (400 MHz, $\mathrm{CDCl}_{3}, \mathrm{rt}$ ): $\delta 2.16$ (s, 3H), 7.30 (s, 1H), 7.36-7.51 (m, 5H), 7.59-7.62 (m, 2H), 7.90-7.92 (m, 1H), 8.00-8.03 (m, 1H); ${ }^{13} \mathrm{C}\left\{{ }^{1} \mathrm{H}\right\} \mathrm{NMR}$ (75 MHz, $\left.\mathrm{CDCl}_{3}, \mathrm{rt}\right): \delta 16.9,121.4,122.8,125.1,125.2,126.2,128.4,128.7,129.0,135.4,138.7$, 147.4, 152.3, 164.5. MS (EI, m/z (relative intensity)): $283\left(\mathrm{M}^{+}, 80\right), 268$ (100), 251 (13), 250 (68), 236 (61), 223 (16), 134 (14), 109 (15). Anal. Calcd for $\mathrm{C}_{16} \mathrm{H}_{13} \mathrm{NS}_{2}$ : C, 67.81; H, 4.62; N, 4.94\%. Found: $\mathrm{C}, 67.91 ; \mathrm{H}, 4.66 ; \mathrm{N}, 4.86 \%$.

\section{(Z)-2-\{2-(p-Methoxyphenyl)-2-Methylthioethenyl\}benzothiazole (3ab)}

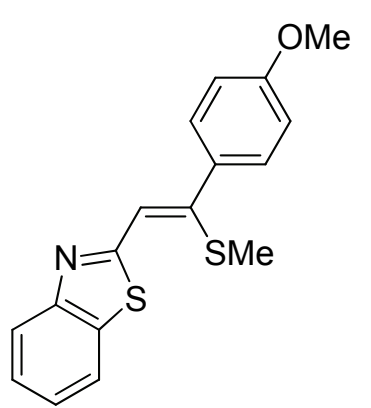

The title compound was obtained in $81 \%$ (253 $\mathrm{mg}, 0.807 \mathrm{mmol})$ as orange solid. $\quad \mathrm{R}_{f}=0.09$ (hexane:ethyl acetate $=20: 1$ ). M.p. $55-56{ }^{\circ} \mathrm{C} . \quad$ FT-IR $\left(\mathrm{KBr}, \mathrm{cm}^{-1}\right)$ : 2957 (w), 2924 (w), 2835 (w), 1603 (s), 1504 (s), 1250 (s), 760 (s). ${ }^{1} \mathrm{H} \mathrm{NMR}\left(400 \mathrm{MHz}, \mathrm{CDCl}_{3}, \mathrm{rt}\right): \delta 2.16(\mathrm{~s}, 3 \mathrm{H}), 3.85(\mathrm{~s}, 3 \mathrm{H})$, 6.94-6.97 (m, 2H), $7.25(\mathrm{~s}, 1 \mathrm{H}), 7.36(\mathrm{td}, J=8.0,1.2 \mathrm{~Hz}, 1 \mathrm{H}), 7.46(\mathrm{td}, J$ $=8.0,1.2 \mathrm{~Hz}, 1 \mathrm{H}), 7.54-7.56(\mathrm{~m}, 2 \mathrm{H}), 7.87-7.89(\mathrm{~m}, 1 \mathrm{H}), 7.97-7.99(\mathrm{~m}$, 
$1 \mathrm{H}) ;{ }^{13} \mathrm{C}\left\{{ }^{1} \mathrm{H}\right\}$ NMR $\left(150 \mathrm{MHz}, \mathrm{CDCl}_{3}, \mathrm{rt}\right): \delta 16.8,55.0,113.9,121.1,122.4,124.0,124.8,125.8$, 129.4, 130.6, 135.1, 146.8, 152.0, 160.1, 164.6. MS (EI, m/z (relative intensity)): $313\left(\mathrm{M}^{+}, 100\right)$, 312 (38), 299 (18), 298 (99), 281 (30), 280 (92), 266 (46), 255 (16), 254 (17), 223 (49). Anal. Calcd for $\mathrm{C}_{17} \mathrm{H}_{15} \mathrm{NOS}_{2}$ : C, 65.14; H, 4.82; N, 4.47\%. Found: C, 65.41; H, 5.13; N, 4.24\%.

(Z)-2-\{2-(p-Methylphenyl)-2-methylthioethenyl\}benzothiazole (3ac)

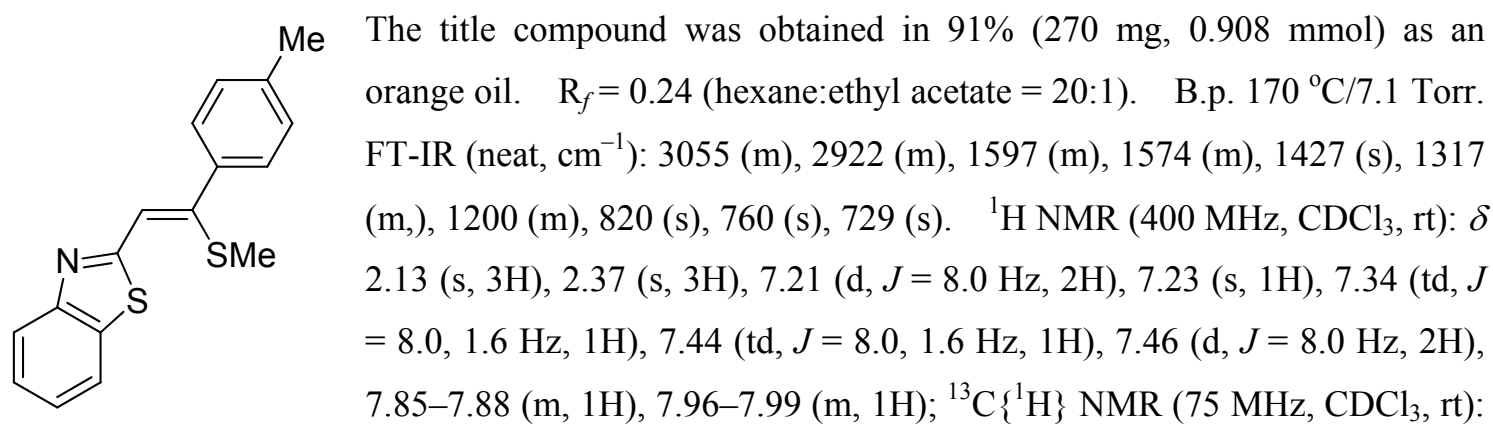
$\delta 17.0,21.3,121.4,122.6,124.5,125.1,126.2,128.3,129.5,135.3,135.7,139.3,147.6,152.2,164.7$. MS (EI, m/z (relative intensity)): 297 (M+, 77), 296 (37), 283 (20), 282 (100), 265 (15), 264 (69), 250 (51), 249 (17), 223 (14), 115 (19). Anal. Calcd for $\mathrm{C}_{17} \mathrm{H}_{15} \mathrm{NS}_{2}$ : C, 68.65; H, 5.08; N, 4.71\%. Found: C, 68.27; H, 4.84; N, 4.53\%.

\section{(Z)-2-[2-Methylthio-2-\{p-(trifluoromethyl)phenyl\}ethenyl]benzothiazole (3ad)}

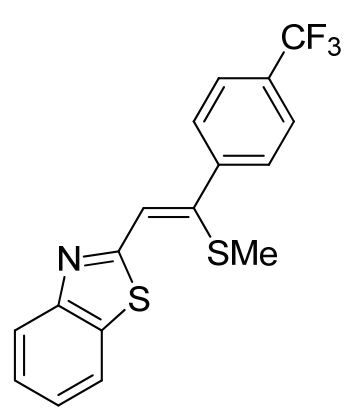

The title compound was obtained in $44 \%$ (77 $\mathrm{mg}, 0.219 \mathrm{mmol})$ as pale yellow solid. $\quad \mathrm{R}_{f}=0.27$ (hexane:ethyl acetate $=20: 1$ ). M.p. $50-52{ }^{\circ} \mathrm{C}$. FT-IR (KBr, cm ${ }^{-1}$ ): 2928 (w), 1614 (m), 1325 (s), 1169 (s), 1121 (s), 723 (s), 540 (s). ${ }^{1} \mathrm{H}$ NMR (300 MHz, $\left.\mathrm{CDCl}_{3}, \mathrm{rt}\right): \delta 2.15$ (s, 3H), 7.34 (s, 3H), $7.40(\mathrm{td}, J=8.1,1.2 \mathrm{~Hz}, 1 \mathrm{H}), 7.50(\mathrm{td}, J=8.1,1.2 \mathrm{~Hz}, 1 \mathrm{H}), 7.72(\mathrm{~s}, 4 \mathrm{H})$, $7.92(\mathrm{~d}, J=8.1 \mathrm{~Hz}, 1 \mathrm{H}), 8.03(\mathrm{~d}, J=8.1 \mathrm{~Hz}, 1 \mathrm{H}) ;{ }^{13} \mathrm{C}\left\{{ }^{1} \mathrm{H}\right\}$ NMR $(150$ $\left.\mathrm{MHz}, \mathrm{CDCl}_{3}, \mathrm{rt}\right): \delta 16.9,121.5,122.9,123.9$ (q, $\left.J=270 \mathrm{~Hz}\right), 125.6,125.8$ (q, $J=3.6 \mathrm{~Hz}), 126.4,126.5,128.7,130.0,130.8$ (q, $J=33 \mathrm{~Hz}) 135.4,142.3,152.0,164.0 ;{ }^{19} \mathrm{~F} \mathrm{NMR}$ (300 MHz, $\left.\mathrm{CDCl}_{3}, \mathrm{rt}\right): 10.8$. MS (EI, m/z (relative intensity)): $351\left(\mathrm{M}^{+}, 78\right), 350$ (24), 337 (18), 336 (100), 318 (50), 304 (50), 223 (12), 109 (14), 108 (12), 69 (13). Anal. Calcd for $\mathrm{C}_{17} \mathrm{H}_{12} \mathrm{~F}_{3} \mathrm{NS}_{2}$ : C, 58.10; H, 3.44; N, 3.99\%. Found: C, 57.70; H, 3.45; N, 3.85\%. 


\section{(Z)-2-\{2-Methylthio-2-(1-naphthyl)ethenyl\}benzothiazole (3ae)}

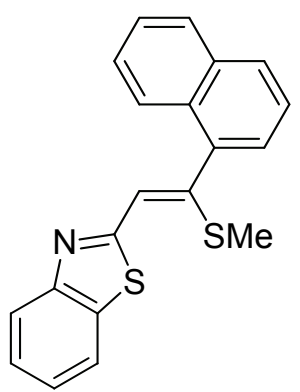

The title compound was obtained in $34 \%$ (56 $\mathrm{mg}, 0.169 \mathrm{mmol}$ ) as orange solid. $\mathrm{R}_{f}=0.45$ (hexane:ethyl acetate $\left.=10: 1\right) . \quad$ M.p. $50-51{ }^{\circ} \mathrm{C} . \quad$ FT-IR $\left(\mathrm{KBr}, \mathrm{cm}^{-}\right.$ $\left.{ }^{1}\right): 3055$ (s), $2922(\mathrm{~s}), 2205$ (w), $1942(\mathrm{w}), 1557$ (m), 1427 (m), 1315 (m), $1117(\mathrm{~m}), 1015$ (m), 937 (w). ${ }^{1} \mathrm{H}$ NMR (600 MHz, $\left.\mathrm{CDCl}_{3}, \mathrm{rt}\right): \delta 1.87(\mathrm{~s}, 3 \mathrm{H})$, $7.08(\mathrm{~s}, 1 \mathrm{H}), 7.38-7.41(\mathrm{~m}, 1 \mathrm{H}), 7.46-7.47(\mathrm{~m}, 1 \mathrm{H}), 7.49-7.55$ (m, 5H), 7.89$7.94(\mathrm{~m}, 3 \mathrm{H}), 8.09(\mathrm{~d}, J=7.8 \mathrm{~Hz}, 1 \mathrm{H}), 8.20-8.22(\mathrm{~m}, 1 \mathrm{H}) ;{ }^{13} \mathrm{C}\left\{{ }^{1} \mathrm{H}\right\} \mathrm{NMR}$ $\left(150 \mathrm{MHz}, \mathrm{CDCl}_{3}, \mathrm{rt}\right): \delta 15.8,121.1,121.4,122.8,124.9,125.1,125.2,126.2$, $126.4,126.6,126.9,128.4,129.0,131.2,133.4,135.2,136.2,148.0,152.8,164.0 . \quad$ MS (EI, m/z (relative intensity)): $333\left(\mathrm{M}^{+}, 75\right), 318$ (100), 300 (30), 286 (80), 152 (23). HRMS (FAB+): Calcd for $\mathrm{C}_{20} \mathrm{H}_{16} \mathrm{NS}_{2}$ : 334.0724 . Found: $334.0716[\mathrm{M}+\mathrm{H}]^{+}$.

\section{(Z)-2-(2-Methylthio-1-octenyl)benzothiazole (3af)}

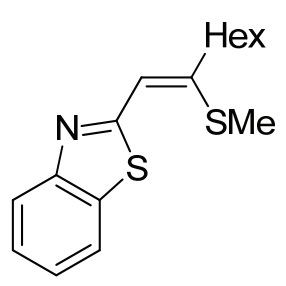

The title compound was obtained in $67 \%(195 \mathrm{mg}, 0.669 \mathrm{mmol})$ as pale yellow solid. $\quad \mathrm{R}_{f}=0.27$ (hexane:ethyl acetate $=20: 1$ ). M.p. $42{ }^{\circ} \mathrm{C} . \quad$ FT-IR $(\mathrm{KBr}$, $\mathrm{cm}^{-1}$ ): 2928 (s), 2853 (s), 1574 (s), 1454 (s), 1427 (s), 1292 (m), 758 (s), 727 (s). ${ }^{1} \mathrm{H}$ NMR (300 MHz, $\left.\mathrm{CDCl}_{3}, \mathrm{rt}\right): \delta 0.90(\mathrm{t}, J=6.9 \mathrm{~Hz}, 3 \mathrm{H}), 1.25-1.40(\mathrm{~m}$, $6 \mathrm{H}$ ), 1.67 (quin, $J=7.5 \mathrm{~Hz}, 2 \mathrm{H}), 2.45(\mathrm{~s}, 3 \mathrm{H}), 2.56(\mathrm{t}, J=7.5 \mathrm{~Hz}, 2 \mathrm{H}), 6.95$ (s, 1H), $7.34(\mathrm{td}, J=8.1,1.2 \mathrm{~Hz}, 1 \mathrm{H}), 7.45(\mathrm{td}, J=8.1,1.2 \mathrm{~Hz}, 1 \mathrm{H}), 7.86$ (d, $J=8.1 \mathrm{~Hz}, 1 \mathrm{H}), 7.98(\mathrm{~d}, J$ $=8.1 \mathrm{~Hz}, 1 \mathrm{H}) ;{ }^{13} \mathrm{C}\left\{{ }^{1} \mathrm{H}\right\} \mathrm{NMR}\left(100 \mathrm{MHz}, \mathrm{CDCl}_{3}, \mathrm{rt}\right): \delta 14.0,14.8,22.5,28.6,28.9,31.6,36.9,120.5$, 121.2, 122.5, 124.6, 125.9, 135.2, 148.6, 152.4, 164.3. MS (EI, m/z (relative intensity)): $291\left(\mathrm{M}^{+}\right.$, 45), 276 (28), 245 (18), 244 (100), 234 (30), 188 (31), 187 (25), 186 (25), 174 (21), 173 (28). Anal. Calcd for $\mathrm{C}_{16} \mathrm{H}_{21} \mathrm{NS}_{2}: \mathrm{C}, 65.93 ; \mathrm{H}, 7.26 ; \mathrm{N}, 4.81 \%$. Found: $\mathrm{C}, 66.30 ; \mathrm{H}, 7.13 ; \mathrm{N}, 4.81 \%$.

\section{(Z)-2-(2-Methylthio-1-hexenyl)benzothiazole (3ag)}

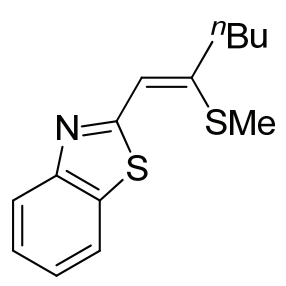

The title compound was obtained in 70\% (184 mg, $0.699 \mathrm{mmol})$ as yellow solid. $\mathrm{R}_{f}=0.17$ (hexane:ethyl acetate $=20: 1$ ). $\quad$ M.p. $50-52{ }^{\circ} \mathrm{C} . \quad$ FT-IR $\left(\mathrm{KBr}, \mathrm{cm}^{-1}\right)$ : 2945 (s), 2112 (w), 1782 (w), 1597 (s), 1015 (m), 748 (s). ${ }^{1} \mathrm{H}$ NMR (600 $\left.\mathrm{MHz}, \mathrm{CDCl}_{3}, \mathrm{rt}\right): \delta 0.96(\mathrm{t}, J=7.2 \mathrm{~Hz}, 3 \mathrm{H}), 1.43(\mathrm{sex}, J=7.2 \mathrm{~Hz}, 2 \mathrm{H}), 1.65$ (quin, $J=7.2 \mathrm{~Hz}, 2 \mathrm{H}), 2.45(\mathrm{~s}, 3 \mathrm{H}), 2.56(\mathrm{t}, J=7.2 \mathrm{~Hz}, 2 \mathrm{H}), 6.97(\mathrm{~s}, 1 \mathrm{H}), 7.34$ (t, $J=7.8 \mathrm{~Hz}, 1 \mathrm{H}), 7.45$ (t, $J=7.8 \mathrm{~Hz}, 1 \mathrm{H}), 7.86(\mathrm{~d}, J=7.8 \mathrm{~Hz}, 1 \mathrm{H}), 7.99$ (d, $J=7.8 \mathrm{~Hz}, 1 \mathrm{H})$; ${ }^{13} \mathrm{C}\left\{{ }^{1} \mathrm{H}\right\}$ NMR $\left(150 \mathrm{MHz}, \mathrm{CDCl}_{3}, \mathrm{rt}\right): \delta 13.9,14.8,22.1,31.1,36.6,120.3,121.3,122.4,124.7$, 126.1, 135.1, 149.3, 151.9, 164.4. MS (EI, m/z (relative intensity)): $263\left(\mathrm{M}^{+}, 82\right), 248$ (44), 234 (35), 230 (19), 216 (100), 188 (21), 187 (35), 186 (37), 174 (36), 173 (40). HRMS (FAB+): Calcd for $\mathrm{C}_{14} \mathrm{H}_{18} \mathrm{NS}_{2}: 264.0881$. Found: $264.0901[\mathrm{M}+\mathrm{H}]^{+}$. 


\section{(Z)-2-(3,3-Dimethyl-2-methylthio-1-butenyl)benzothiazole (3ah)}

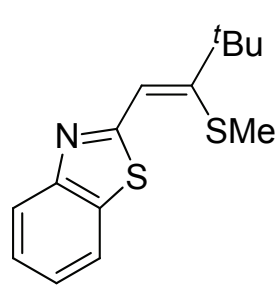

The title compound was obtained in $60 \%(79 \mathrm{mg}, 0.300 \mathrm{mmol})$ as white solid. $\mathrm{R}_{f}=0.36$ (hexane:ethyl acetate $\left.=20: 1\right) . \quad$ M.p. $246-247{ }^{\circ} \mathrm{C} . \quad$ FT-IR $\left(\mathrm{KBr}, \mathrm{cm}^{-}\right.$ 1): $3057(\mathrm{w}), 2965(\mathrm{~m}), 2290(\mathrm{w}), 1454(\mathrm{~m}), 1427(\mathrm{~m}), 970(\mathrm{w}), 883(\mathrm{w}), 760$ (s), $729(\mathrm{~m}) .{ }^{1} \mathrm{H}$ NMR (300 MHz, $\left.\mathrm{CDCl}_{3}, \mathrm{rt}\right): \delta 1.31(\mathrm{~s}, 9 \mathrm{H}), 2.35(\mathrm{~s}, 3 \mathrm{H})$, $7.39(\mathrm{td}, J=7.8,1.2 \mathrm{~Hz}, 1 \mathrm{H}), 7.43(\mathrm{~s}, 1 \mathrm{H}), 7.47(\mathrm{td}, J=7.8,1.2 \mathrm{~Hz}, 1 \mathrm{H}) 7.87-$ $7.90(\mathrm{~m}, 1 \mathrm{H}), 7.98-8.02(\mathrm{~m}, 1 \mathrm{H}) ;{ }^{13} \mathrm{C}\left\{{ }^{1} \mathrm{H}\right\} \operatorname{NMR}\left(75 \mathrm{MHz}, \mathrm{CDCl}_{3}, \mathrm{rt}\right): \delta 21.5,29.1,41.3,121.35$, 121.37, 121.38, 122.8, 125.4, 126.1, 128.4, 157.4, 165.4. MS (EI, m/z (relative intensity)): 263 $\left(\mathrm{M}^{+}, 100\right), 248$ (82), 230 (37), 201 (21), 200 (30), 192 (21), 174 (23). Anal. Calcd for $\mathrm{C}_{14} \mathrm{H}_{17} \mathrm{NS}_{2}$ : C, 63.83; H, 6.50; N, 5.32\%. Found: C, 63.92; H, 6.72; N, 5.04\%.

\section{(Z)-2-(3,3-Diethoxy-2-methylthio-1-propenyl)benzothiazole (3ai)}

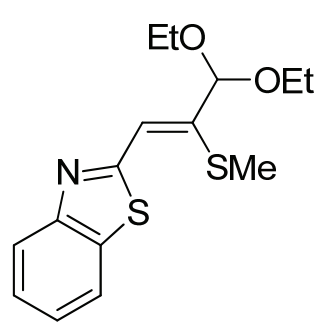

The title compound was isolated in $75 \%(232 \mathrm{mg}, 0.750 \mathrm{mmol})$ as a pale yellow oil. $\mathrm{R}_{f}=0.25$ (hexane:ethyl acetate $=10: 1$ ). B.p. $200{ }^{\circ} \mathrm{C} / 7.2$ Torr. FT-IR (neat, $\mathrm{cm}^{-1}$ ): 3057 (w), 2974 (m), 1578 (m), 1454 (m), 1429 (m), 1126 (s), 1059 (s), 760 (s). ${ }^{1} \mathrm{H}$ NMR (400 MHz, $\mathrm{CDCl}_{3}, \mathrm{rt}$ ): $\delta 1.28$ (t, $J=7.2 \mathrm{~Hz}$, $6 \mathrm{H}), 2.61(\mathrm{~s}, 3 \mathrm{H}), 3.55-3.74(\mathrm{~m}, 4 \mathrm{H}), 5.19(\mathrm{~s}, 1 \mathrm{H}), 7.38(\mathrm{td}, J=7.2,1.2 \mathrm{~Hz}$, 1H), 7.40 (s, 1H), 7.49 (td, $J=7.2 \mathrm{~Hz}, 1.2 \mathrm{~Hz}, 1 \mathrm{H}), 7.88-7.91$ (m, 1H), $8.03-$ $8.05(\mathrm{~m}, 1 \mathrm{H}) ;{ }^{13} \mathrm{C}\left\{{ }^{1} \mathrm{H}\right\} \mathrm{NMR}\left(150 \mathrm{MHz}, \mathrm{CDCl}_{3}, \mathrm{rt}\right): \delta 15.1,15.6,61.9,102.3,121.4,122.86,122.94$, 125.2, 126.2, 135.6, 142.9, 152.2, 163.6. MS (EI, m/z (relative intensity)): 309 (M+1 4.6), 280 (83), 233 (55), 103 (53), 75 (100). Anal. Calcd for $\mathrm{C}_{15} \mathrm{H}_{19} \mathrm{NO}_{2} \mathrm{~S}_{2}$ : C, 58.22; H, 6.19; N, 4.53\%. Anal. Calcd for $\mathrm{C}_{15} \mathrm{H}_{19} \mathrm{NO}_{2} \mathrm{~S}_{2}$ : C, 58.22; H, 6.19; N, 4.53\%. Found: C, 58.40; H, 6.23; N, 4.45\%.

\section{(Z)-2-\{2-(p-Ethynylphenyl)-2-methylthioethenyl\}benzothiazole (3aj)}

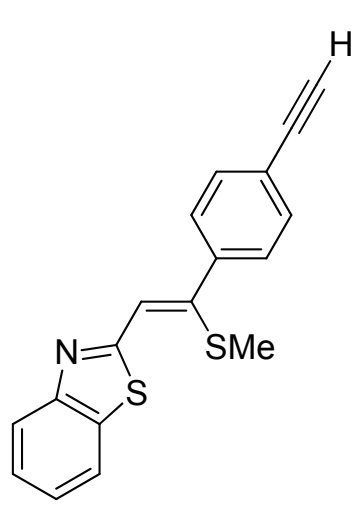

The title compound was obtained in $52 \%(80 \mathrm{mg}, 0.260 \mathrm{mmol})$ as pale yellow solid. $\quad \mathrm{R}_{f}=0.20$ (hexane:ethyl acetate $=20: 1$ ). $\quad$ M.p. $128-130{ }^{\circ} \mathrm{C}$. FT-IR (KBr, cm ${ }^{-1}$ ): 3264 (s), 2920 (m), 1954 (w), 1425 (m), 851 (s), 752 (s). ${ }^{1} \mathrm{H}$ NMR (400 MHz, $\left.\mathrm{CDCl}_{3}, \mathrm{rt}\right): \delta 2.17(\mathrm{~s}, 3 \mathrm{H}), 3.18(\mathrm{~s}, 1 \mathrm{H}), 7.35$ (s, $1 \mathrm{H}), 7.40(\mathrm{td}, J=8.0,1.2 \mathrm{~Hz}, 1 \mathrm{H}), 7.50(\mathrm{td}, J=8.0,1.2 \mathrm{~Hz}, 1 \mathrm{H}), 7.58-$ $7.59(\mathrm{~m}, 4 \mathrm{H}), 7.90-7.92(\mathrm{~m}, 1 \mathrm{H}), 8.02-8.04(\mathrm{~m}, 1 \mathrm{H}) ;{ }^{13} \mathrm{C}\left\{{ }^{1} \mathrm{H}\right\} \mathrm{NMR}(100$ $\left.\mathrm{MHz}, \mathrm{CDCl}_{3}, \mathrm{rt}\right): \delta 16.9,78.7,83.0,121.3,122.79,122.82,125.3,125.9$, $126.2,128.2,132.4,135.4,139.0,146.0,152.2,164.2$. MS $(\mathrm{EI}, \mathrm{m} / \mathrm{z}$ (relative intensity)): $307\left(\mathrm{M}^{+}, 84\right), 292$ (100), 274 (68), 260 (62). Anal.

Calcd for $\mathrm{C}_{18} \mathrm{H}_{13} \mathrm{NS}_{2}$ : C, 70.32; H, 4.26; N, 4.56\%. Found: C, 70.00; H, 3.86; N, 4.38\%. 


\section{(Z)-2-(2-Methylthio-1-hepten-6-ynyl)benzothiazole (3ak)}

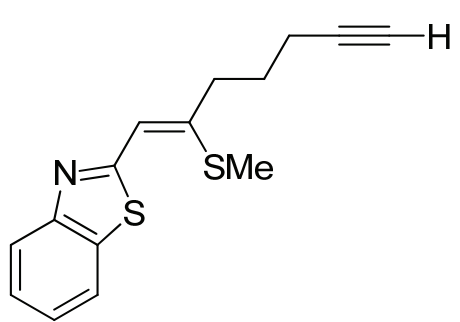

The title compound was obtained in $30 \%(82 \mathrm{mg}, 0.300 \mathrm{mmol})$ as pale yellow solid. $\mathrm{R}_{f}=0.06$ (hexane:ethyl acetate $=20: 1$ ). M.p. 30-31 ${ }^{\circ} \mathrm{C}$. FT-IR (KBr, cm ${ }^{-1}$ ): 2955 (s), 1601 (s), 1454 (s), 1314 (m), $1144(\mathrm{~m}), 758(\mathrm{~s}) .{ }^{1} \mathrm{H}$ NMR $\left(400 \mathrm{MHz}, \mathrm{CDCl}_{3}, \mathrm{rt}\right): \delta 1.90$ (quin, $J=7.2 \mathrm{~Hz}, 2 \mathrm{H}), 2.02(\mathrm{t}, J=2.8 \mathrm{~Hz}, 1 \mathrm{H}), 2.32(\mathrm{td}, J=7.2$, $2.8 \mathrm{~Hz}, 2 \mathrm{H}), 2.47$ (s, 3H), 2.71 (t, $J=7.2 \mathrm{~Hz}, 2 \mathrm{H}), 6.99$ (s, 1H), $7.34(\mathrm{td}, J=7.6,1.2 \mathrm{~Hz}, 1 \mathrm{H}), 7.45(\mathrm{td}, J=7.6,1.2 \mathrm{~Hz}, 1 \mathrm{H}), 7.86-7.88(\mathrm{~m}, 1 \mathrm{H}), 7.98-8.00(\mathrm{~m}, 1 \mathrm{H})$; ${ }^{13} \mathrm{C}\left\{{ }^{1} \mathrm{H}\right\}$ NMR $\left(75 \mathrm{MHz}, \mathrm{CDCl}_{3}, \mathrm{rt}\right): \delta 14.9,17.6,27.6,35.5,69.3,83.4,121.29,121.33,122.6$, 124.8, 126.1, 135.2, 147.4, 152.3, 164.1. MS (EI, m/z (relative intensity)): $273\left(\mathrm{M}^{+}, 40\right), 258(45)$, 240 (23), 234 (100), 226 (76), 224 (24), 187 (73), 186 (46), 173 (30), 109 (25). Anal. Calcd for $\mathrm{C}_{15} \mathrm{H}_{15} \mathrm{NS}_{2}: \mathrm{C}, 65.89 ; \mathrm{H}, 5.53 ; \mathrm{N}, 5.12 \%$. Found: C, 66.22; H, 5.43; N, 5.03\%.

\section{(Z)-2-\{(2-Tetrahydropyranylidene)methyl\}benzothiazole (3al')}

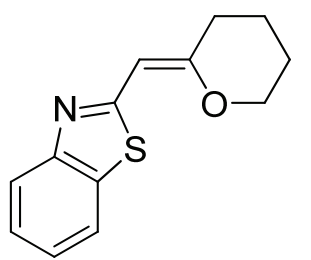

The title compound was obtained in $74 \%(171 \mathrm{mg}, 0.739 \mathrm{mmol})$ as pale yellow solid. $\mathrm{R}_{f}=0.09$ (hexane:ethyl acetate $=10: 1$ ). M.p. $118-119{ }^{\circ} \mathrm{C}$. FT-IR (KBr, cm ${ }^{-1}$ ): 3055 (m), 2953 (s), 2862 (m), 1942 (w), 1697 (s), 1587 (m), 1306 (s), 1094 (m), 1067 (s). ${ }^{1} \mathrm{H}$ NMR (400 MHz, $\left.\mathrm{CDCl}_{3}, \mathrm{rt}\right): \delta 1.81-$ 1.92 (m, 4H), 2.50 (t, $J=6.0 \mathrm{~Hz}, 2 \mathrm{H}), 4.27$ (t, $J=5.6 \mathrm{~Hz}, 2 \mathrm{H}), 6.09$ (s, 1H), $7.28(\mathrm{td}, J=7.6,1.2 \mathrm{~Hz}, 1 \mathrm{H}), 7.40(\mathrm{td}, J=7.6,1.2 \mathrm{~Hz}, 1 \mathrm{H}), 7.81-7.83(\mathrm{~m}, 1 \mathrm{H}), 7.90-7.92(\mathrm{~m}, 1 \mathrm{H})$; ${ }^{13} \mathrm{C}\left\{{ }^{1} \mathrm{H}\right\}$ NMR $\left(150 \mathrm{MHz}, \mathrm{CDCl}_{3}, \mathrm{rt}\right): \delta 20.9,24.1,29.2,68.9,102.7,121.1,121.8,124.0,125.7$, 134.9, 151.8, 162.4, 163.7. MS (EI, m/z (relative intensity)): $231\left(\mathrm{M}^{+}, 87\right), 230$ (34), 203 (21), 202 (100). HRMS (FAB+): Calcd for $\mathrm{C}_{13} \mathrm{H}_{15} \mathrm{NOS}: 232.0796$. Found: $232.0807[\mathrm{M}+\mathrm{H}]^{+}$.

\section{(Z)-2-(2-Phenyl-2-phenylthioethenyl)benzothiazole (3ba)}

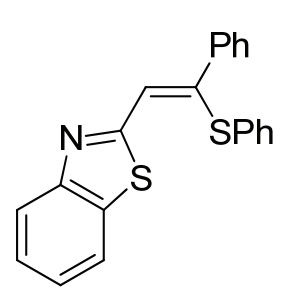

The title compound was obtained in $82 \%$ (283 $\mathrm{mg}, 0.819 \mathrm{mmol})$ as orange solid. $\mathrm{R}_{f}=0.13$ (hexane:ethyl acetate $\left.=20: 1\right) . \quad$ M.p. $134-135^{\circ} \mathrm{C} . \quad$ FT-IR $(\mathrm{KBr}, \mathrm{cm}$

$\left.{ }^{1}\right): 3057(\mathrm{w}), 3017(\mathrm{w}), 1479(\mathrm{~m}), 1204(\mathrm{~m}), 762(\mathrm{~s}), 691(\mathrm{~s}) .{ }^{1} \mathrm{H}$ NMR (300 $\left.\mathrm{MHz}, \mathrm{CDCl}_{3}, \mathrm{rt}\right): \delta 7.08-7.17(\mathrm{~m}, 3 \mathrm{H}), 7.25-7.30(\mathrm{~m}, 5 \mathrm{H}), 7.38(\mathrm{td}, J=8.1,1.2$ $\mathrm{Hz}, 1 \mathrm{H}), 7.49(\mathrm{td}, J=8.1,1.2 \mathrm{~Hz}, 1 \mathrm{H}), 7.67-7.70(\mathrm{~m}, 2 \mathrm{H}), 7.73(\mathrm{~s}, 1 \mathrm{H}), 7.86(\mathrm{~d}$, $J=8.1 \mathrm{~Hz}, 1 \mathrm{H}), 8.05(\mathrm{~d}, J=8.1 \mathrm{~Hz}, 1 \mathrm{H}) ;{ }^{13} \mathrm{C}\left\{{ }^{1} \mathrm{H}\right\} \mathrm{NMR}\left(150 \mathrm{MHz}, \mathrm{CDCl}_{3}, \mathrm{rt}\right): \delta 121.4,123.0$, $125.6,126.3,126.9,128.3,128.4,129.0,129.1,129.4,130.0,133.2,135.5,138.8,142.7,152.3$, 164.5. MS (EI, m/z (relative intensity)): $345\left(\mathrm{M}^{+}, 100\right), 344$ (73), 312 (46), 269 (15), 268 (83), 236 (44), 210 (31), 121 (19), 109 (17), 77 (15). Anal. Calcd for $\mathrm{C}_{21} \mathrm{H}_{15} \mathrm{NS}_{2}$ : C, 73.01; H, 4.38; N, 4.05\%. Found: C, 73.01; H, 4.25; N, 3.99 . 


\section{(Z)-2-(2-Methylthio-2-phenylethenyl)naphtho[1,2-d] thiazole (3ca)}

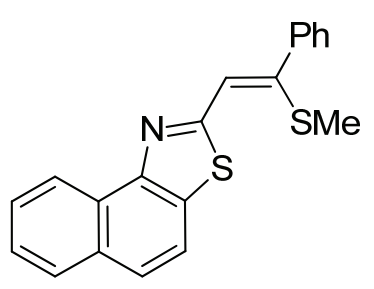

The title compound was obtained in $75 \%(125 \mathrm{mg}, 0.375 \mathrm{mmol})$ as a brown viscous oil. $\mathrm{R}_{f}=0.40$ (hexane:ethyl acetate $=20: 1$ ). B.p. 210 ${ }^{\circ} \mathrm{C} / 7.1$ Torr. FT-IR (neat, $\mathrm{cm}^{-1}$ ): 3053 (s), 3024 (m), 2922 (m), 2826 (w), 1954 (w), 1746 (w), 1568 (m), 1487 (s), 1215 (s), 1078 (s), 1024 (m), 905 (m), 806 (s). $\quad{ }^{1} \mathrm{H}$ NMR (400 MHz, $\left.\mathrm{CDCl}_{3}, \mathrm{rt}\right): \delta 2.18$ (s, 3H), 7.41-7.48 (m, 4H), $7.60(\mathrm{td}, J=8.4,1.2 \mathrm{~Hz}, 1 \mathrm{H}), 7.65-7.67(\mathrm{~m}, 2 \mathrm{H}), 7.70(\mathrm{td}, J=8.4 \mathrm{~Hz}, 1.2 \mathrm{~Hz}, 1 \mathrm{H}), 7.80(\mathrm{~d}$, $J=8.8 \mathrm{~Hz}, 1 \mathrm{H}), 7.92(\mathrm{~d}, J=8.8 \mathrm{~Hz}, 1 \mathrm{H}), 7.95-7.97(\mathrm{~m}, 1 \mathrm{H}), 8.87-8.89(\mathrm{~m}, 1 \mathrm{H}) ;{ }^{13} \mathrm{C}\left\{{ }^{1} \mathrm{H}\right\} \mathrm{NMR}$ $\left(150 \mathrm{MHz}, \mathrm{CDCl}_{3}, \mathrm{rt}\right): \delta 16.9,118.8,123.8,125.1,125.9,126.0,126.8,128.0,128.27,128.35,128.6$, 128.8, 132.0, 132.1, 138.7, 145.8, 148.7, 163.5. MS (EI, m/z (relative intensity)): $333\left(\mathrm{M}^{+}, 100\right)$, 318 (50), 300 (80), 207 (39), 159 (38). HRMS (FAB+): Calcd for $\mathrm{C}_{20} \mathrm{H}_{16} \mathrm{NS}_{2}$ : 334.0724. Found: $334.0720[\mathrm{M}+\mathrm{H}]^{+}$.

\section{(Z)-2-(2-Methylthio-2-phenylethenyl)-4-phenylthiazole (3da)}<smiles>CO/C(=C\c1nc(-c2ccccc2)cs1)c1ccccc1</smiles>

The title compound was obtained in $79 \%(122 \mathrm{mg}, 0.394 \mathrm{mmol})$ as a brown viscous oil. $\quad \mathrm{R}_{f}=0.37$ (hexane:ethyl acetate $=20: 1$ ). B.p. $190{ }^{\circ} \mathrm{C} / 6.9$ Torr. FT-IR (neat, $\mathrm{cm}^{-1}$ ): 3059 (m), 3024 (m), 2922 (m), 1952 (w), 1587 (m), 1574 (m), 1470 (s), 1445 (s), 1072 (m), 1059 (m). ${ }^{1} \mathrm{H}$ NMR (600 MHz, CDCl ${ }_{3}$, rt): $\delta 2.15(\mathrm{~s}, 3 \mathrm{H}), 7.33(\mathrm{~s}, 1 \mathrm{H}), 7.35-7.41(\mathrm{~m}, 2 \mathrm{H}), 7.42-7.46(\mathrm{~m}, 4 \mathrm{H}), 7.54(\mathrm{~d}, J=1.2 \mathrm{~Hz}, 1 \mathrm{H})$, 7.59-7.62 (m, 2H), 7.94-7.97 (m, 2H); ${ }^{13} \mathrm{C}\left\{{ }^{1} \mathrm{H}\right\}$ NMR (150 MHz, $\left.\mathrm{CDCl}_{3}, \mathrm{rt}\right): \delta 16.7,113.6,124.7$, $126.4,128.1,128.3,128.6,128.65,128.69,134.3,138.7,143.8,154.7,164.2$. MS (EI, m/z (relative intensity)): $309\left(\mathrm{M}^{+}, 61\right), 294$ (61), 276 (100), 134 (51). Anal. Calcd for $\mathrm{C}_{18} \mathrm{H}_{16} \mathrm{NS}_{2}$ : $\mathrm{C}$, 69.86; H, 4.89; N, 4.53\%. Found: C, 70.12; H, 5.13; N, 4.23.

\section{(Z)-2-(2-Methylthio-2-phenylethenyl)thiazole (3ea)}

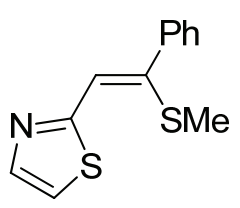

The title compound was obtained in $57 \%(66 \mathrm{mg}, 0.283 \mathrm{mmol})$ as an orange oil. $\mathrm{R}_{f}=0.23$ (hexane:ethyl acetate $=10: 1$ ). B.p. $205{ }^{\circ} \mathrm{C} / 6.8$ Torr. FT-IR (neat, $\left.\mathrm{cm}^{-1}\right): 3111(\mathrm{w}), 3057(\mathrm{w}), 2963(\mathrm{w}), 2207(\mathrm{w}), 1734(\mathrm{w}), 1572(\mathrm{w}), 1491(\mathrm{~m})$, 1470 (m), 1445 (m), 1142 (m), 1092 (m), 876 (w), 760 (s). ${ }^{1} \mathrm{H}$ NMR (400 MHz, $\left.\mathrm{CDCl}_{3}, \mathrm{rt}\right): \delta 2.12(\mathrm{~s}, 3 \mathrm{H}), 7.25(\mathrm{~s}, 1 \mathrm{H}), 7.37-7.45(\mathrm{~m}, 4 \mathrm{H}), 7.56-7.58(\mathrm{~m}, 2 \mathrm{H}), 7.87(\mathrm{~d}, J=4.8 \mathrm{~Hz}$, $1 \mathrm{H}) ;{ }^{13} \mathrm{C}\left\{{ }^{1} \mathrm{H}\right\}$ NMR $\left(150 \mathrm{MHz}, \mathrm{CDCl}_{3}, \mathrm{rt}\right): \delta 16.7,119.4,124.1,127.1,128.4,128.7,128.8,138.6$, 141.7, 164.6. MS (EI, m/z (relative intensity)): $233\left(\mathrm{M}^{+}, 88\right), 232$ (94), 217 (90), 200 (100), 186 (92). HRMS (FAB+): Calcd for $\mathrm{C}_{12} \mathrm{H}_{12} \mathrm{NS}_{2}$ : 234.0411. Found: $234.0397[\mathrm{M}+\mathrm{H}]^{+}$. 


\section{(Z)-2-(2-Methylthio-2-phenylethenyl)benzoxazole (3fa)}

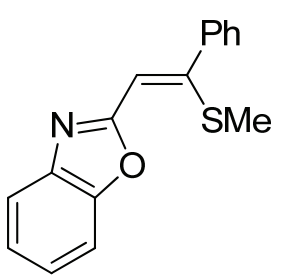

The title compound was obtained in $43 \%(115 \mathrm{mg}, 0.430 \mathrm{mmol})$ as a pale yellow oil. $\mathrm{R}_{f}=0.12$ (hexane:ethyl acetate $=20: 1$ ). B.p. $160{ }^{\circ} \mathrm{C} / 5.7$ Torr. FT-IR (neat, $\mathrm{cm}^{-1}$ ): 3019 (m), 2955 (s), 2928 (s), 2859 (s), 1601 (s), 1576 (s), 1495 (m), 1454 (s), 1377 (w), 1314 (m), 1142 (m). ${ }^{1} \mathrm{H}$ NMR (300 MHz, $\left.\mathrm{CDCl}_{3}, \mathrm{rt}\right): \delta 1.90(\mathrm{~s}, 3 \mathrm{H}), 6.38(\mathrm{~s}, 1 \mathrm{H}), 7.08(\mathrm{~s}, 1 \mathrm{H}), 7.13-7.16(\mathrm{~m}, 2 \mathrm{H}), 7.22-$ $7.27(\mathrm{~m}, 4 \mathrm{H}), 7.32-7.35(\mathrm{~m}, 1 \mathrm{H}), 7.62-7.65(\mathrm{~m}, 1 \mathrm{H}) ;{ }^{13} \mathrm{C}\left\{{ }^{1} \mathrm{H}\right\} \mathrm{NMR}\left(75 \mathrm{MHz}, \mathrm{CDCl}_{3}, \mathrm{rt}\right): \delta 16.6$, $110.2,111.7,120.0,124.3,124.9,128.3,128.6,128.8,138.8,142.3,149.7,153.8,161.5$. MS (EI, m/z (relative intensity)): $267\left(\mathrm{M}^{+}, 100\right), 266$ (55), 220 (42), 252 (27), 234 (24). HRMS (FAB+): Calcd for $\mathrm{C}_{16} \mathrm{H}_{14} \mathrm{NOS}$ : 268.0796. Found: $268.0786[\mathrm{M}+\mathrm{H}]^{+}$.

\section{(Z)-2-(2-Methylthio-2-phenylethenyl)benzothiophene (3ga)}

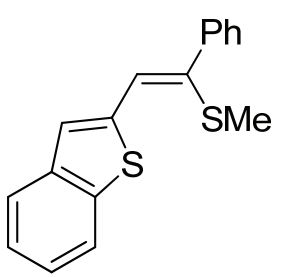

The reaction was conducted on $1 \mathrm{mmol}$ scale. The title compound was obtained in $12 \%$ (34 mg, $0.120 \mathrm{mmol}$ ) as white solid. $\mathrm{R}_{f}=0.44$ (hexane:ethyl acetate $=20: 1) . \quad$ M.p. $81{ }^{\circ} \mathrm{C} . \quad$ FT-IR $\left(\mathrm{KBr}, \mathrm{cm}^{-1}\right): 3046(\mathrm{w}), 2990(\mathrm{w}), 2922$ (m), 1427 (m), 1310 (w), 746 (s). ${ }^{1} \mathrm{H}$ NMR (600 MHz, $\left.\mathrm{CDCl}_{3}, \mathrm{rt}\right): \delta 2.14$ (s, $3 \mathrm{H}), 7.13$ (s, 1H), 7.31-7.37 (m, 3H), 7.42-7.45 (m, 3H), 7.63 (d, $J=7.8 \mathrm{~Hz}$, 2H), $7.73(\mathrm{~d}, J=7.8 \mathrm{~Hz}, 1 \mathrm{H}), 7.83(\mathrm{~d}, J=7.8 \mathrm{~Hz}, 1 \mathrm{H}) ;{ }^{13} \mathrm{C}\left\{{ }^{1} \mathrm{H}\right\} \mathrm{NMR}\left(150 \mathrm{MHz}, \mathrm{CDCl}_{3}, \mathrm{rt}\right): \delta 16.8$, 122.1, 123.2, 124.3, 124.7, 125.7, 126.7, 128.17, 128.17, 128.6, 138.2, 138.6, 139.8, 140.6, 140.9. MS (EI, m/z (relative intensity)): $282\left(\mathrm{M}^{+}, 100\right), 268$ (18), 267 (96), 266 (14), 235 (24), 234 (89), 221 (10), 202 (13), 189 (11), 117 (25). Anal. Calcd for $\mathrm{C}_{17} \mathrm{H}_{14} \mathrm{~S}_{2}$ : C, 72.30; H, 5.00\%. Found: C, 72.45; H, 4.98\%.

\section{Negishi Coupling of Sulfide 3aa: Synthesis of 2-(2,2-Diphenylethenyl)benzothiazole (4)}
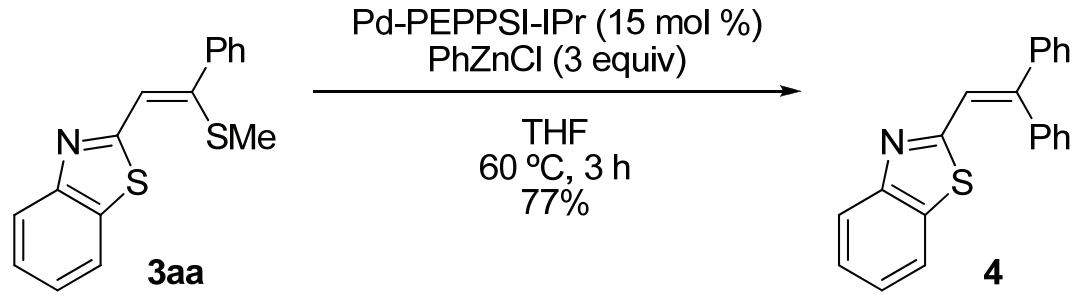

Pd-PEPPSI-IPr (20 mg, $0.030 \mathrm{mmol})$ and 3aa $(57 \mathrm{mg}, 0.20 \mathrm{mmol})$ were placed in a 20-mL Schlenk tube under argon. THF $(3 \mathrm{~mL})$ and $\mathrm{PhZnCl}(0.15 \mathrm{M}$ THF solution, $4.0 \mathrm{~mL}, 0.60 \mathrm{mmol})$ were added. The reaction mixture was stirred at $60{ }^{\circ} \mathrm{C}$ for $3 \mathrm{~h}$. The mixture was poured into saturated $\mathrm{NH}_{4} \mathrm{Cl}$ aqueous solution $(10 \mathrm{~mL})$ and extracted with ethyl acetate $(10 \mathrm{~mL} \times 3)$. The combined organic layers were dried over anhydrous sodium sulfate and concentrated in vacuo. Purification by column chromatography on silica gel (hexane:ethyl acetate $=20: 1$ ) provided 2-(2,2-diphenylethenyl)benzothiazole (4, $48 \mathrm{mg}, 0.153 \mathrm{mmol}, 77 \%)$ as yellow solid. 
$\mathrm{R}_{f}=0.37$ (hexane:ethyl acetate $=20: 1$ ). $\quad$ M.p. 104-105 ${ }^{\circ} \mathrm{C} . \quad$ FT-IR $\left(\mathrm{KBr}, \mathrm{cm}^{-1}\right): 3057(\mathrm{w}), 3030$ (w), 1740 (w), 1597 (w), 1491 (m), 1445 (m), 1431 (m), 1350 (m), 1317 (m), 1256 (w), 874 (w). ${ }^{1} \mathrm{H}$ NMR (300 MHz, $\left.\mathrm{CDCl}_{3}, \mathrm{rt}\right): \delta 7.28(\mathrm{td}, J=8.0,0.8 \mathrm{~Hz}, 1 \mathrm{H}), 7.33-7.37(\mathrm{~m}, 5 \mathrm{H}), 7.39-7.45(\mathrm{~m}$, $3 \mathrm{H}), 7.54-7.57(\mathrm{~m}, 3 \mathrm{H}), 7.63(\mathrm{~d}, J=8.0 \mathrm{~Hz}, 1 \mathrm{H}), 7.67(\mathrm{~s}, 1 \mathrm{H}), 7.98(\mathrm{~d}, J=8.0 \mathrm{~Hz}, 1 \mathrm{H}) ;{ }^{13} \mathrm{C}\left\{{ }^{1} \mathrm{H}\right\}$ NMR (75 MHz, $\left.\mathrm{CDCl}_{3}, \mathrm{rt}\right): \delta 121.1,122.3,122.7,125.1,126.0,127.37,127.38,128.5,128.9,129.0$, 129.5, 130.0, 135.5, 137.9, 140.4, 149.3, 152.1. MS (EI, m/z (relative intensity)): $313\left(\mathrm{M}^{+}, 29\right)$, 312 (100), 235 (19), 206 (50). Anal. Calcd for $\mathrm{C}_{21} \mathrm{H}_{15} \mathrm{NS}$ : C, 80.48; H, 4.82; N, 4.47\%. HRMS $(\mathrm{FAB}+)$ : Calcd for $\mathrm{C}_{21} \mathrm{H}_{15} \mathrm{NS}$ : 313.0925 . Found: $313.0897\left[\mathrm{M}^{+}\right]$.

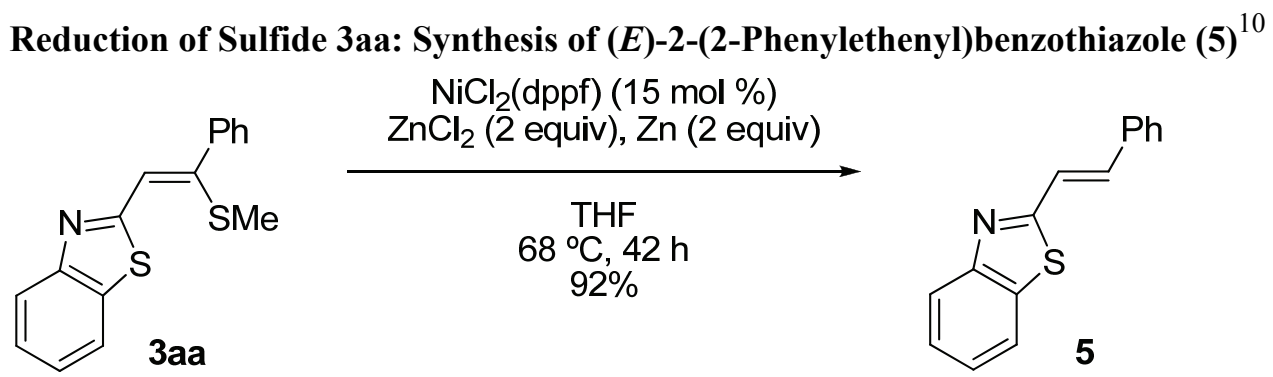

In a $20-\mathrm{mL}$ Schlenk tube, sulfide 3aa $(102 \mathrm{mg}, 0.36 \mathrm{mmol}), \mathrm{NiCl}_{2}(\mathrm{dppf})(37 \mathrm{mg}, 0.054 \mathrm{mmol})$, $\mathrm{ZnCl}_{2}$ (1 M THF solution, $0.72 \mathrm{~mL}, 0.72 \mathrm{mmol}$ ), and $\mathrm{Zn}$ powder (47 $\mathrm{mg}, 0.72 \mathrm{mmol}$ ) were placed under argon. The reaction mixture was stirred at reflux for $42 \mathrm{~h}$. The mixture was filtered through a pad of silica gel. The filtrate was evaporated to yield a crude oil. Purification by column chromatography on silica gel (hexane:ethyl acetate $=20: 1$ ) provided (E)-2-(2-phenylethenyl)benzothiazole $(\mathbf{5}, 79 \mathrm{mg}, 0.333 \mathrm{mmol}, 92 \%)$ as an orange oil. The spectroscopic data of $\mathbf{5}$ can be found in the literature. ${ }^{11}$

${ }^{1} \mathrm{H}$ NMR $\left(300 \mathrm{MHz}, \mathrm{CDCl}_{3}, \mathrm{rt}\right): \delta 7.37-7.52(\mathrm{~m}, 7 \mathrm{H}), 7.57-7.61(\mathrm{~m}, 2 \mathrm{H}), 7.86(\mathrm{~d}, J=7.8 \mathrm{~Hz}, 1 \mathrm{H})$, $8.00(\mathrm{~d}, J=7.8 \mathrm{~Hz}, 1 \mathrm{H})$.

\section{Oxidation of Sulfide 3aa: Synthesis of (Z)-2-(2-Methylsulfinyl-2-phenylethenyl)benzothiazole} (6)
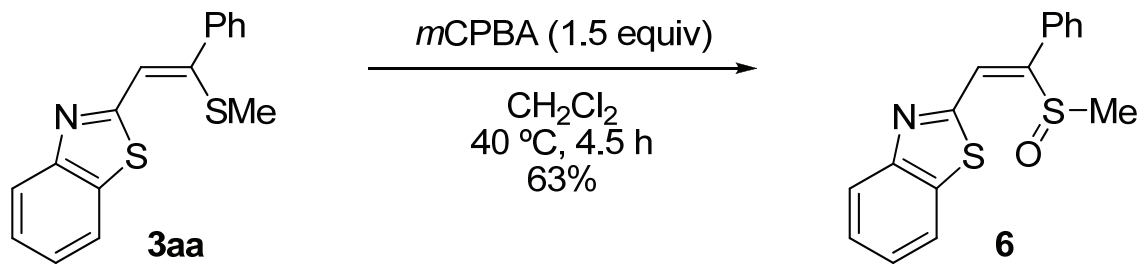

In a 20 -mL Schlenk tube, sulfide $3 \mathbf{a a}(283 \mathrm{mg}, 1.0 \mathrm{mmol})$ was dissolved in dichloromethane (10 $\mathrm{mL}$ ). $m$-Chloroperoxybenzoic acid $(77 \%, 336 \mathrm{mg}, 1.5 \mathrm{mmol})$ was added portionwise to the solution. The reaction mixture was stirred at $40{ }^{\circ} \mathrm{C}$ for $4.5 \mathrm{~h}$. The mixture was cooled to room temperature and then poured into saturated $\mathrm{NaHCO}_{3}$ aqueous solution $(10 \mathrm{~mL})$. The product was 
extracted with dichloromethane $(10 \mathrm{~mL} \times 3)$. The combined organic layers were dried over anhydrous sodium sulfate and concentrated in vacuo. Purification by column chromatography on silica gel (hexane:ethyl acetate $=5: 1)$ afforded sulfoxide $6(190 \mathrm{mg}, 0.635 \mathrm{mmol}, 63 \%)$ as yellow solid.

$\mathrm{R}_{f}=0.07$ (hexane:ethyl acetate $=5: 1$ ). M.p. $160-161^{\circ} \mathrm{C} . \quad$ FT-IR $\left(\mathrm{KBr}, \mathrm{cm}^{-1}\right): 3044(\mathrm{~m}), 3036(\mathrm{~m})$, 2073 (w), 1782 (w), 1583 (s), 1493 (s), 1325 (s), 1040 (s). ${ }^{1}$ H NMR (400 MHz, DMSO- $d_{6}$, rt): $\delta$ $2.92(\mathrm{~s}, 3 \mathrm{H}), 7.47-7.54(\mathrm{~m}, 5 \mathrm{H}), 7.58-7.63(\mathrm{~m}, 3 \mathrm{H}), 8.11-8.13(\mathrm{~m}, 1 \mathrm{H}), 8.18-8.20(\mathrm{~m}, 1 \mathrm{H}) ;{ }^{13} \mathrm{C}\left\{{ }^{1} \mathrm{H}\right\}$ NMR (150 MHz, DMSO- $d_{6}$, rt): $\delta 34.5,122.5,123.4,125.2,126.2,127.0,128.0,129.3,129.7,132.5$, 135.9, 152.9, 154.4, 160.9. $\mathrm{MS}\left(\mathrm{FAB}^{+}\right): 300\left([\mathrm{M}+\mathrm{H}]^{+}\right)$. Anal. Calcd for $\mathrm{C}_{16} \mathrm{H}_{13} \mathrm{NOS}_{2}: \mathrm{C}, 64.18$; H, 4.38; N, 4.68\%. Found: C, 64.16; H, 4.27; N, 4.53\%.

\section{$\begin{array}{llllll}\text { Allylation } & \text { of } & \text { Sulfoxide } & 6: & \text { Synthesis } & \text { of }\end{array}$}

2-(1-Methylthio-1-phenyl-1,4-pentadien-2-yl)benzothiazole $(7)^{12}$

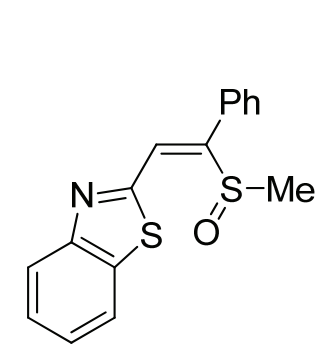

6

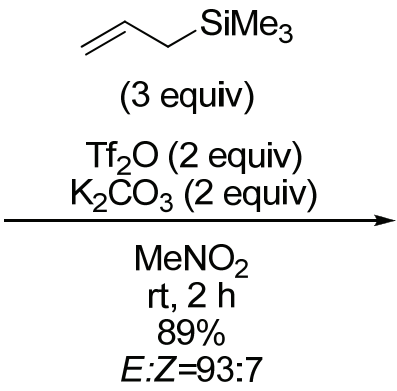

$E: Z=93: 7$

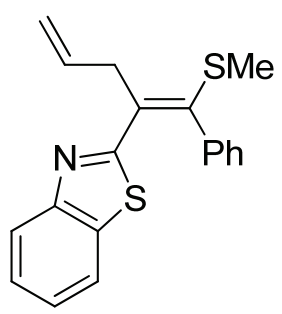

$(E)-7$

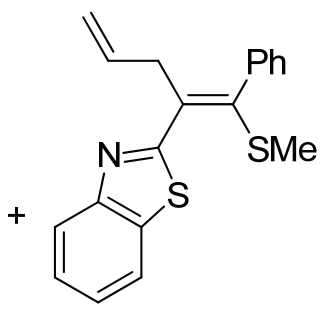

(Z)-7

A mixture of nitromethane $(1 \mathrm{~mL}), 6(90 \mathrm{mg}, 0.30 \mathrm{mmol})$, potassium carbonate $(83 \mathrm{mg}, 0.60 \mathrm{mmol})$, and allyltrimethylsilane $(0.14 \mathrm{~mL}, 0.90 \mathrm{mmol})$ was placed in a $20-\mathrm{mL}$ Schlenk tube under an atmosphere of argon. Trifluoromethanesulfonic anhydride $(169 \mathrm{mg}, 0.60 \mathrm{mmol})$ was added to the solution at $0{ }^{\circ} \mathrm{C}$, and the mixture was stirred at room temperature for $2 \mathrm{~h}$. The mixture was poured into saturated $\mathrm{NaHCO}_{3}$ aqueous solution $(10 \mathrm{~mL})$ and the product was extracted with dichloromethane $(10 \mathrm{~mL} \times 3)$. The combined organic layers were dried over anhydrous sodium sulfate and concentrated under reduced pressure. Purification by silica gel column chromatography (hexane:ethyl acetate $=20: 1)$ afforded $7(86 \mathrm{mg}, 0.266 \mathrm{mmol}, 89 \%, E / Z=93 / 7)$ as a pale yellow oil. $\mathrm{R}_{f}=0.45$ (hexane:ethyl acetate $=20: 1$ ). B.p. $170{ }^{\circ} \mathrm{C} / 7.5$ Torr. $\mathrm{MS}(\mathrm{EI}, \mathrm{m} / \mathrm{z}$ (relative intensity)): 323 (M+, 21), 308 (100), 276 (39), 274 (25), 121 (33). Anal. Calcd for $\mathrm{C}_{19} \mathrm{H}_{17} \mathrm{NS}_{2}$ : C, 70.55; H, 5.30 ; N, 4.33\%. Found: C, 70.56; H, 5.13; N, 4.28\%.

Stereoisomers $(E / Z)-7$ were separated by gel-permeation chromatography $\left(\mathrm{CHCl}_{3}\right)$. 


\section{(E)-2-(1-Methylthio-1-phenyl-1,4-pentadien-2-yl)benzothiazole ( $(E)-7)$}

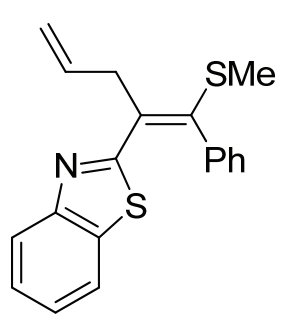

FT-IR (neat, $\mathrm{cm}^{-1}$ ): 3075 (w), 2922 (w), 2363 (w), 1636 (w), 1570 (m), 1485 (m), 1431 (s), 1315 (w), 910 (m), 758 (s). ${ }^{1} \mathrm{H}$ NMR (600 MHz, $\left.\mathrm{CDCl}_{3}, \mathrm{rt}\right): \delta$ $1.86(\mathrm{~s}, 3 \mathrm{H}), 4.00(\mathrm{dd}, J=6.0,3.6 \mathrm{~Hz}, 2 \mathrm{H}), 5.07$ (dd, $J=10,1.8 \mathrm{~Hz}, 1 \mathrm{H}), 5.21$ $(\mathrm{dtd}, J=17,3.6,1.8 \mathrm{~Hz}, 1 \mathrm{H}), 5.97-6.03(\mathrm{~m}, 1 \mathrm{H}), 7.22(\mathrm{t}, J=7.8 \mathrm{~Hz}, 1 \mathrm{H})$, $7.29-7.31(\mathrm{~m}, 2 \mathrm{H}), 7.36$ (t, $J=7.8 \mathrm{~Hz}, 1 \mathrm{H}), 7.41-7.43$ (m, 3H), 7.56 (d, $J=7.8$ $\mathrm{Hz}, 1 \mathrm{H}), 7.92(\mathrm{~d}, J=7.8 \mathrm{~Hz}, 1 \mathrm{H}) ;{ }^{13} \mathrm{C}\left\{{ }^{1} \mathrm{H}\right\}$ NMR $\left(150 \mathrm{MHz}, \mathrm{CDCl}_{3}, \mathrm{rt}\right): \delta 15.4$, 38.2, 115.6, 120.8, 122.8, 124.6, 125.6, 128.9, 129.1, 129.9, 130.4, 134.6, 135.8, 136.6, 146.1, 152.0, 167.0.

\section{(Z)-2-(1-Methylthio-1-phenyl-1,4-pentadien-2-yl)benzothiazole ((Z)-7)}

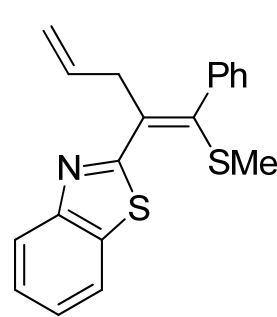

FT-IR (neat, $\mathrm{cm}^{-1}$ ): 3075 (w), 2922 (w), 2363 (w), 1717 (w), 1431 (m), 1315 (w), 760 (m). ${ }^{1} \mathrm{H}$ NMR (600 MHz, $\left.\mathrm{CDCl}_{3}, \mathrm{rt}\right): \delta 1.84$ (s, 3H), 3.30 (dd, $J=$ 6.0, $1.2 \mathrm{~Hz}, 2 \mathrm{H}), 4.91-4.95(\mathrm{~m}, J=2 \mathrm{H}), 5.77-5.83(\mathrm{~m}, 1 \mathrm{H}), 7.31(\mathrm{~d}, J=7.8 \mathrm{~Hz}$, 2H), 7.35-7.39 (m, 2H), 7.43-7.49 (m, 3H), 7.89 (d, $J=7.8 \mathrm{~Hz}, 1 \mathrm{H}), 8.09$ (d, $J$ $=7.8 \mathrm{~Hz}, 1 \mathrm{H}) ;{ }^{13} \mathrm{C}\left\{{ }^{1} \mathrm{H}\right\}$ NMR $\left(150 \mathrm{MHz}, \mathrm{CDCl}_{3}, \mathrm{rt}\right): \delta 16.5,39.3,116.2,121.3$, $123.1,125.0,125.9,128.0,128.1,128.5,128.8,135.2,135.7,137.8,145.0$,

$152.5,166.8$. 


\section{Copies of ${ }^{1} \mathrm{H}$ and ${ }^{13}$ C NMR Charts of the New Compounds}
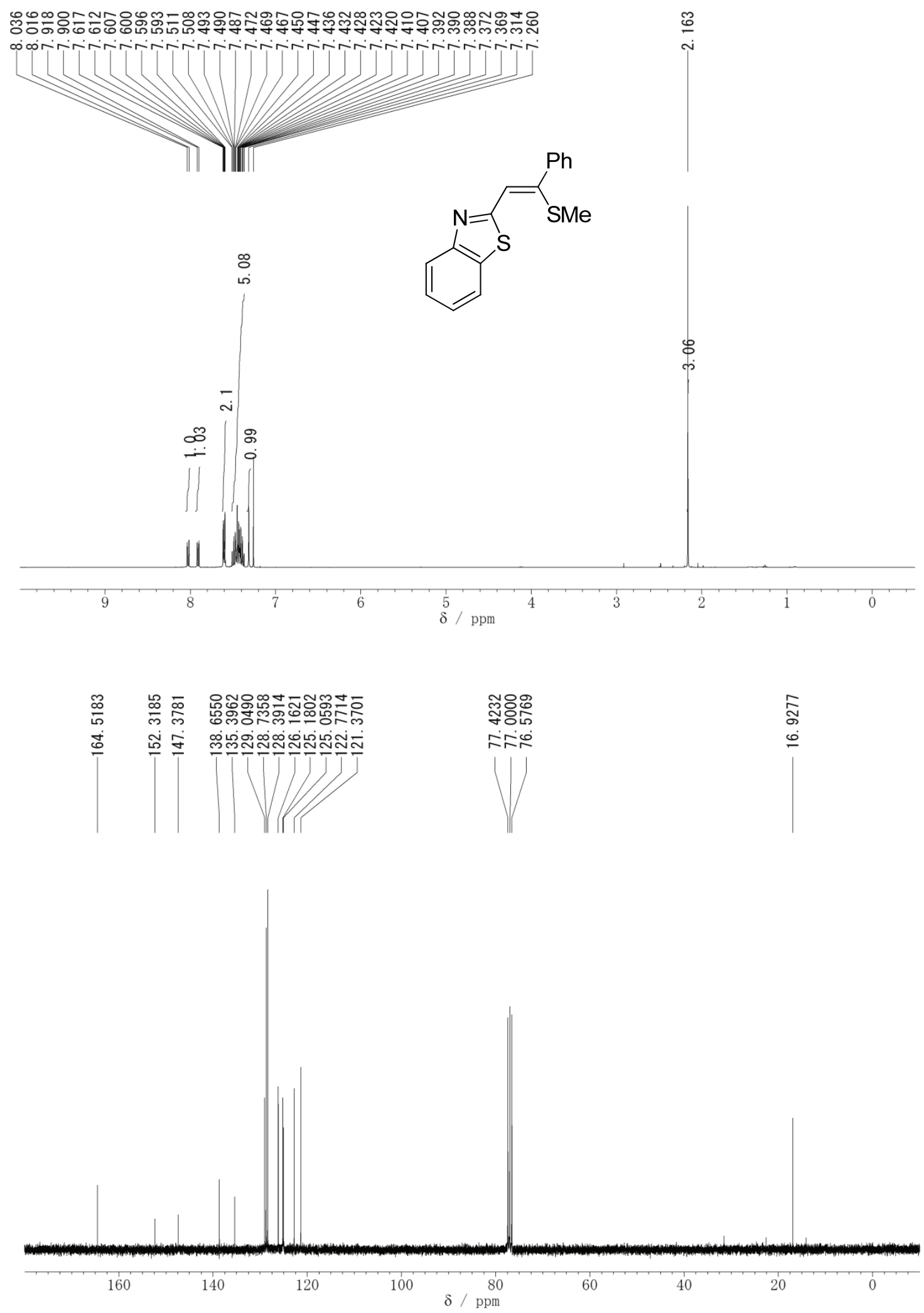

${ }^{1} \mathrm{H}$ NMR $(400 \mathrm{MHz})$ and ${ }^{13} \mathrm{C}\left\{{ }^{1} \mathrm{H}\right\}$ NMR (75 MHz) spectra of 3aa (rt, $\left.\mathrm{CDCl}_{3}\right)$. 

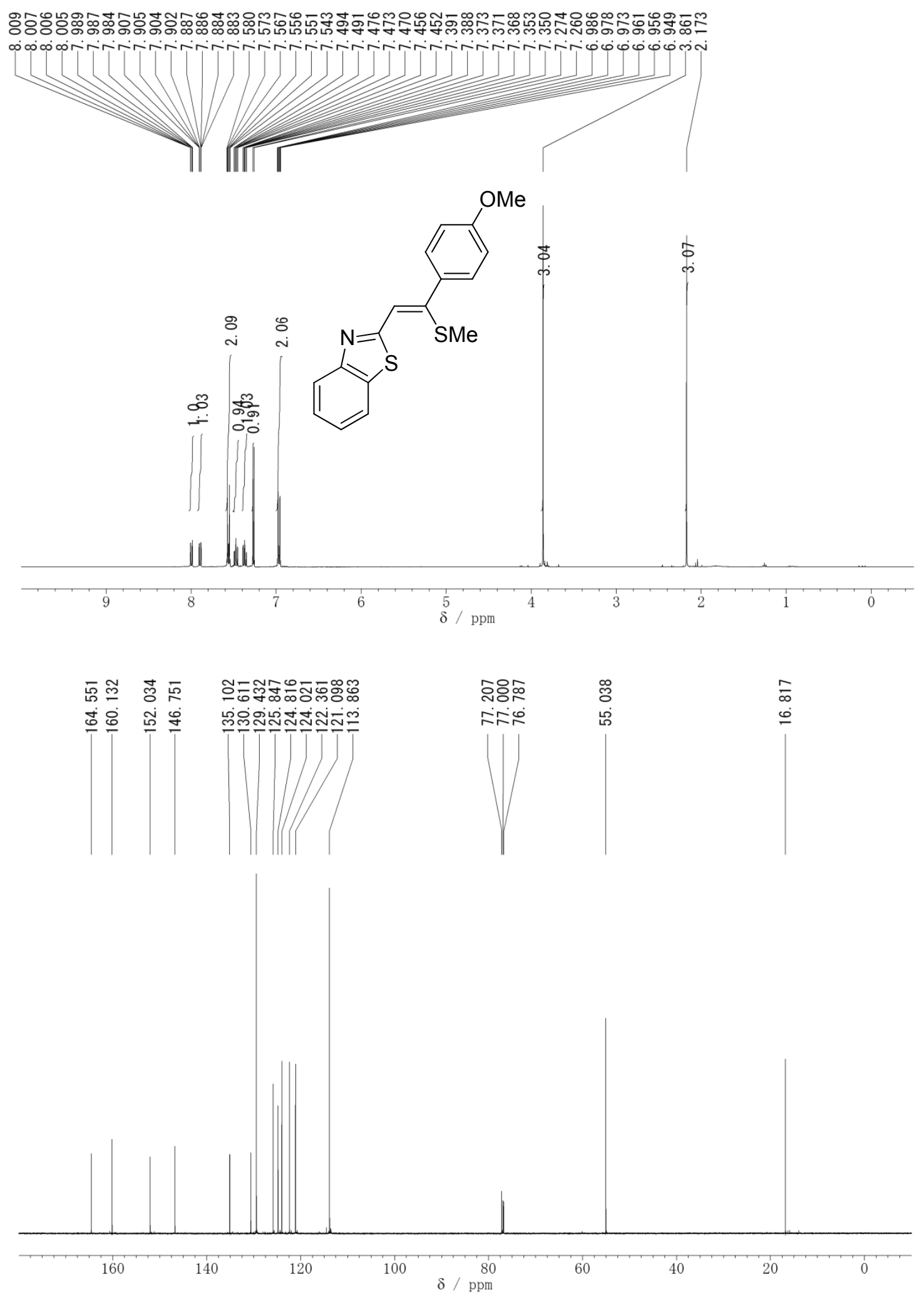

${ }^{1} \mathrm{H}$ NMR (400 MHz) and ${ }^{13} \mathrm{C}\left\{{ }^{1} \mathrm{H}\right\}$ NMR $(150 \mathrm{MHz})$ spectra of $\mathbf{3 a b}\left(\mathrm{rt}, \mathrm{CDCl}_{3}\right)$. 


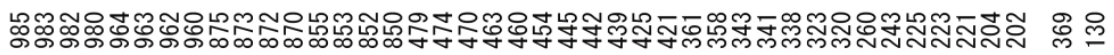

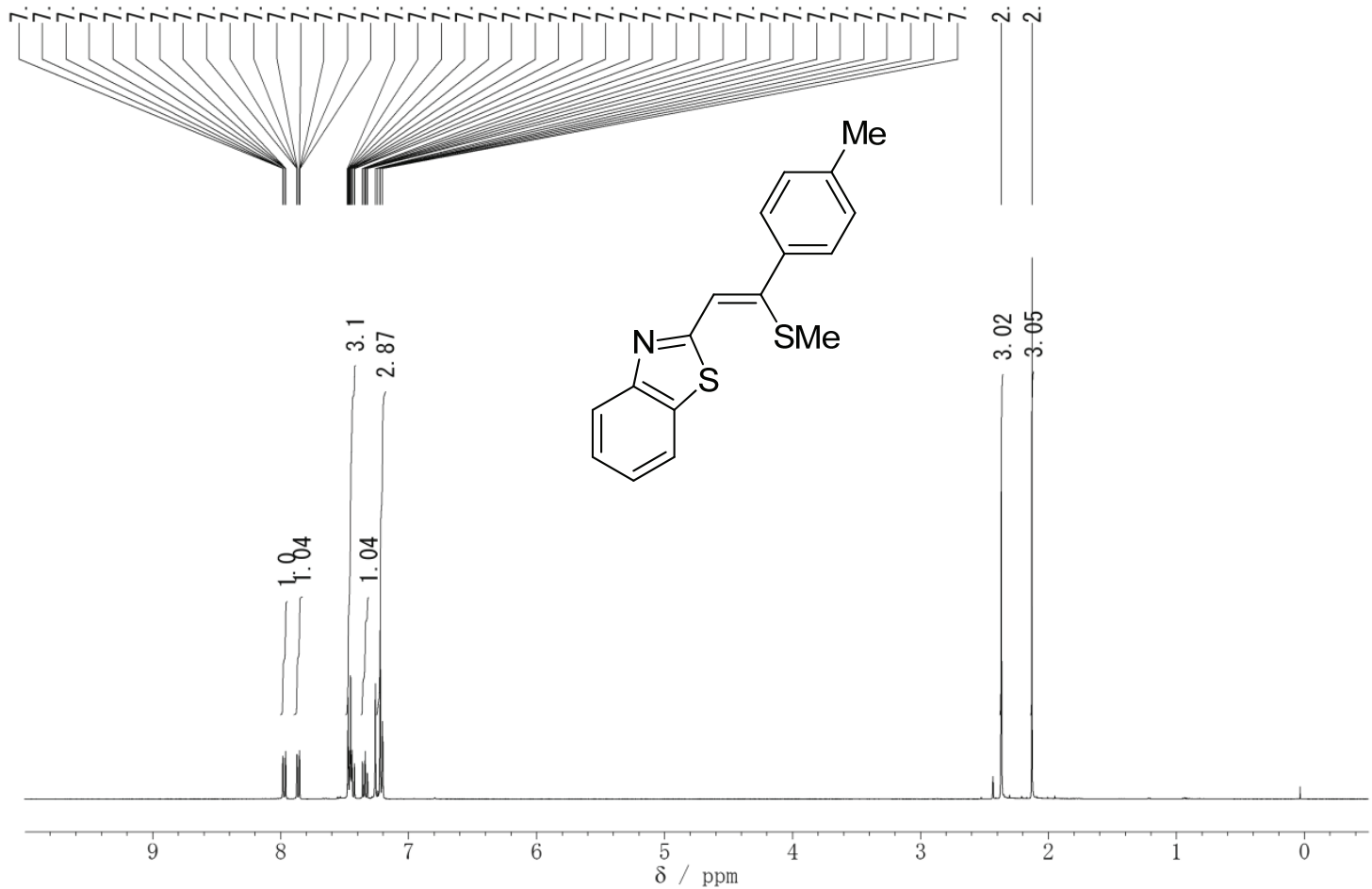

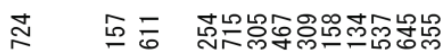

ชูำ

สั

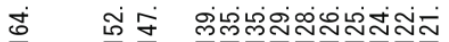

सi⿱宀⿻三丨口

$\dot{4}=$
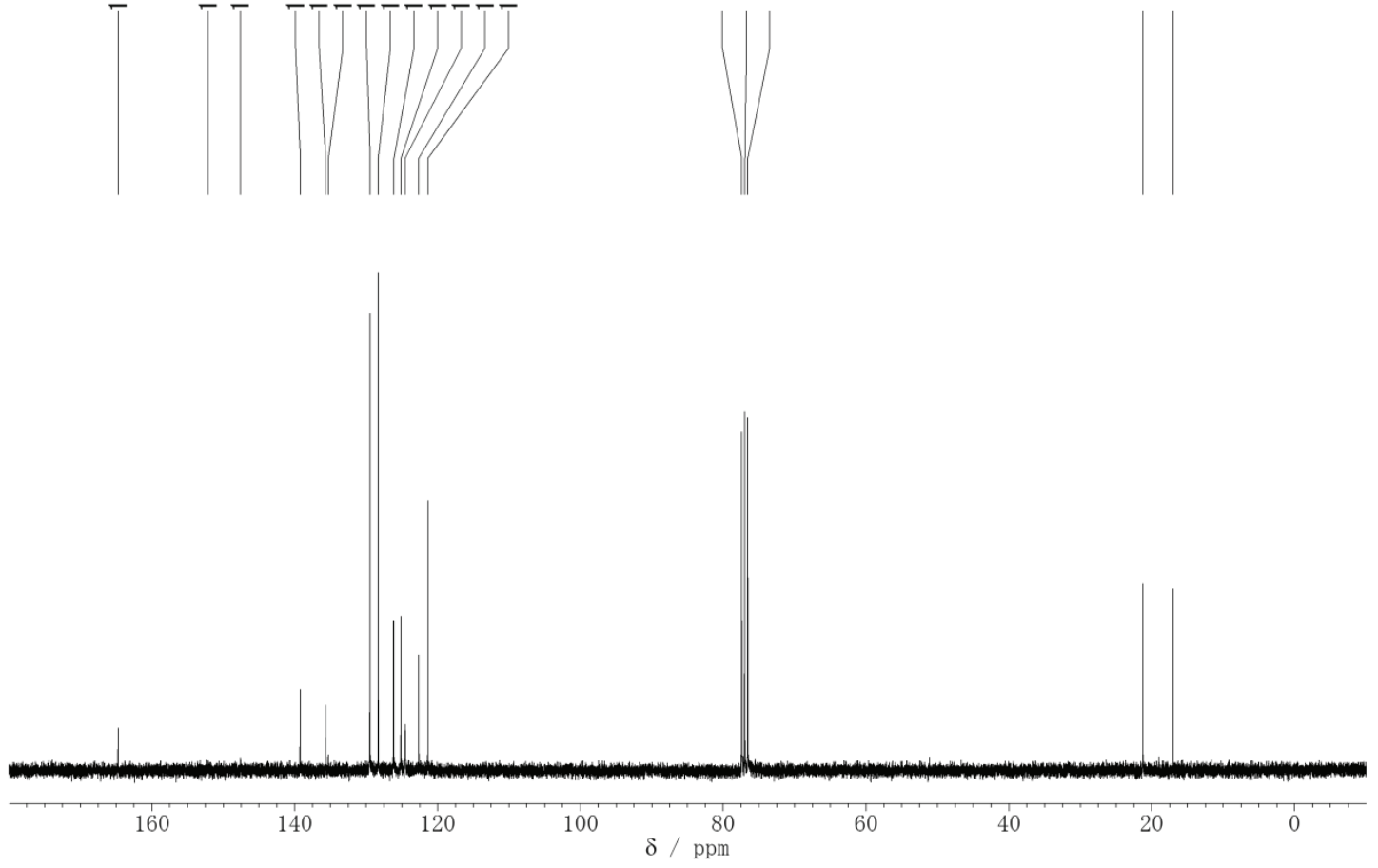

${ }^{1} \mathrm{H}$ NMR $(400 \mathrm{MHz})$ and ${ }^{13} \mathrm{C}\{1 \mathrm{H}\}$ NMR $(75 \mathrm{MHz})$ spectra of $\mathbf{3 a c}\left(\mathrm{rt}, \mathrm{CDCl}_{3}\right)$. 


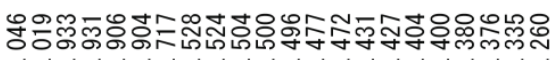

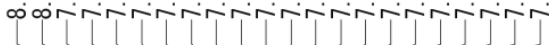

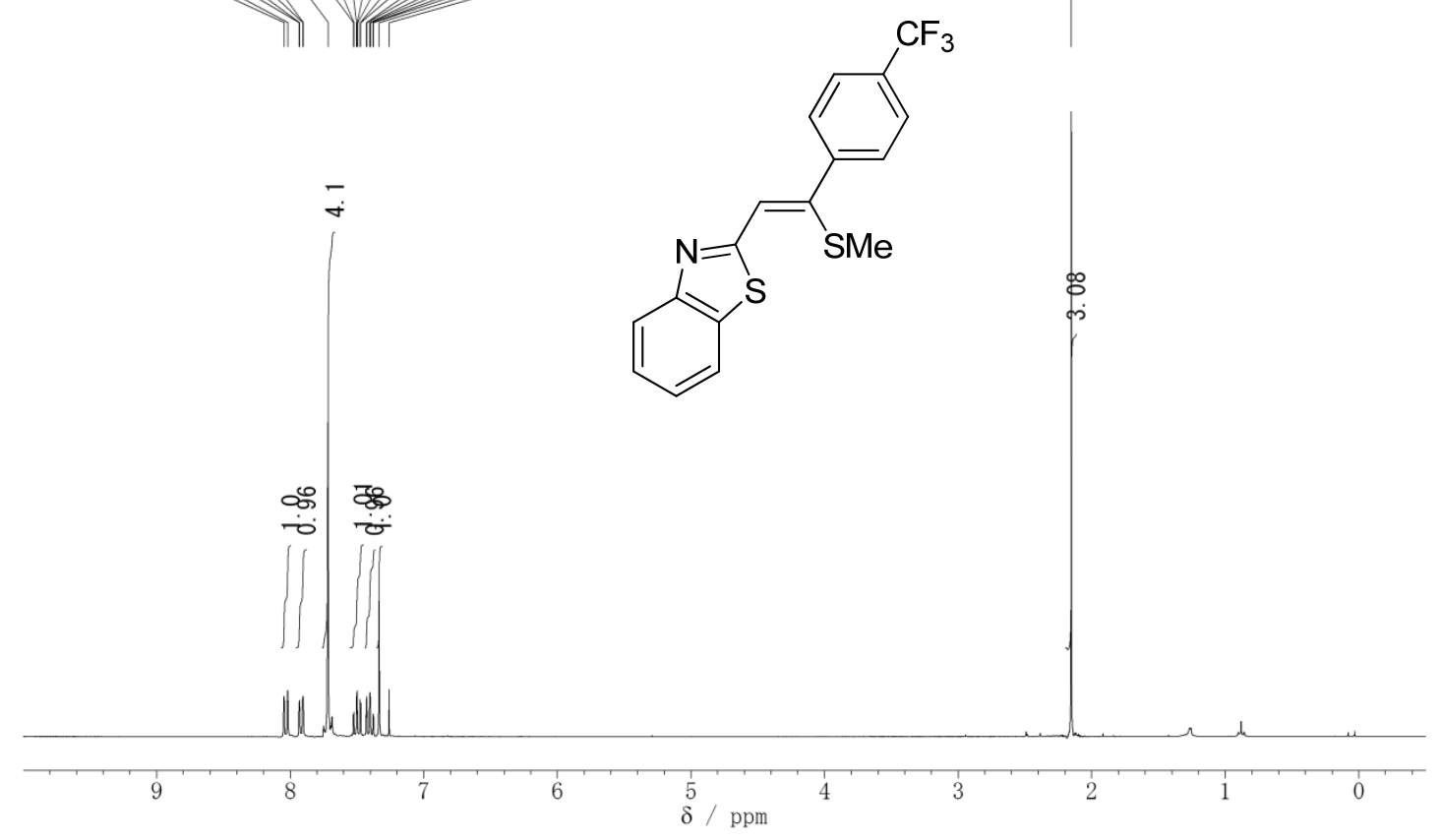

M Nifi
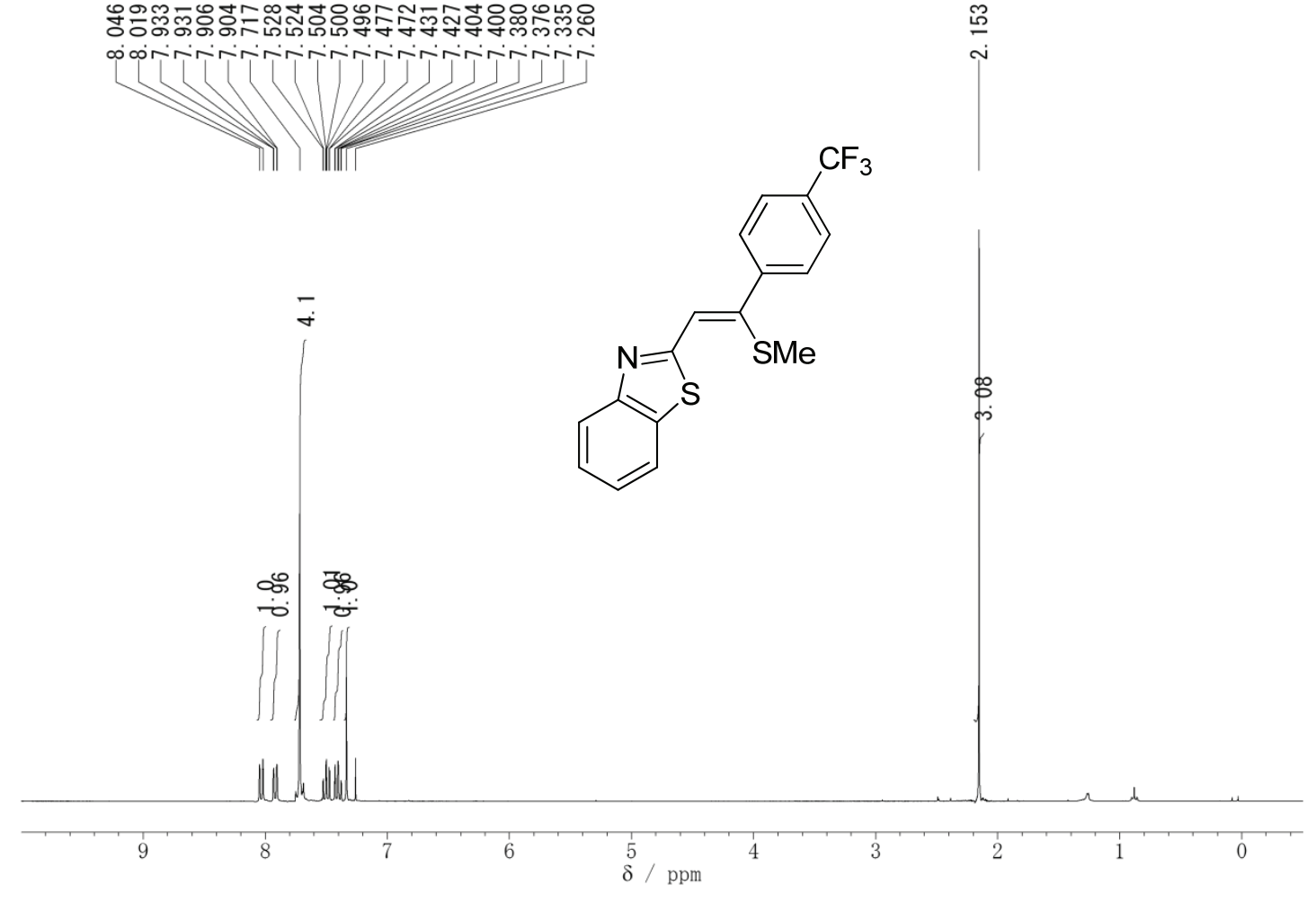

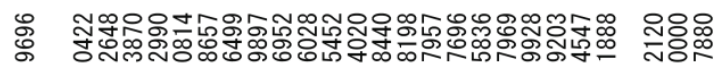

๕
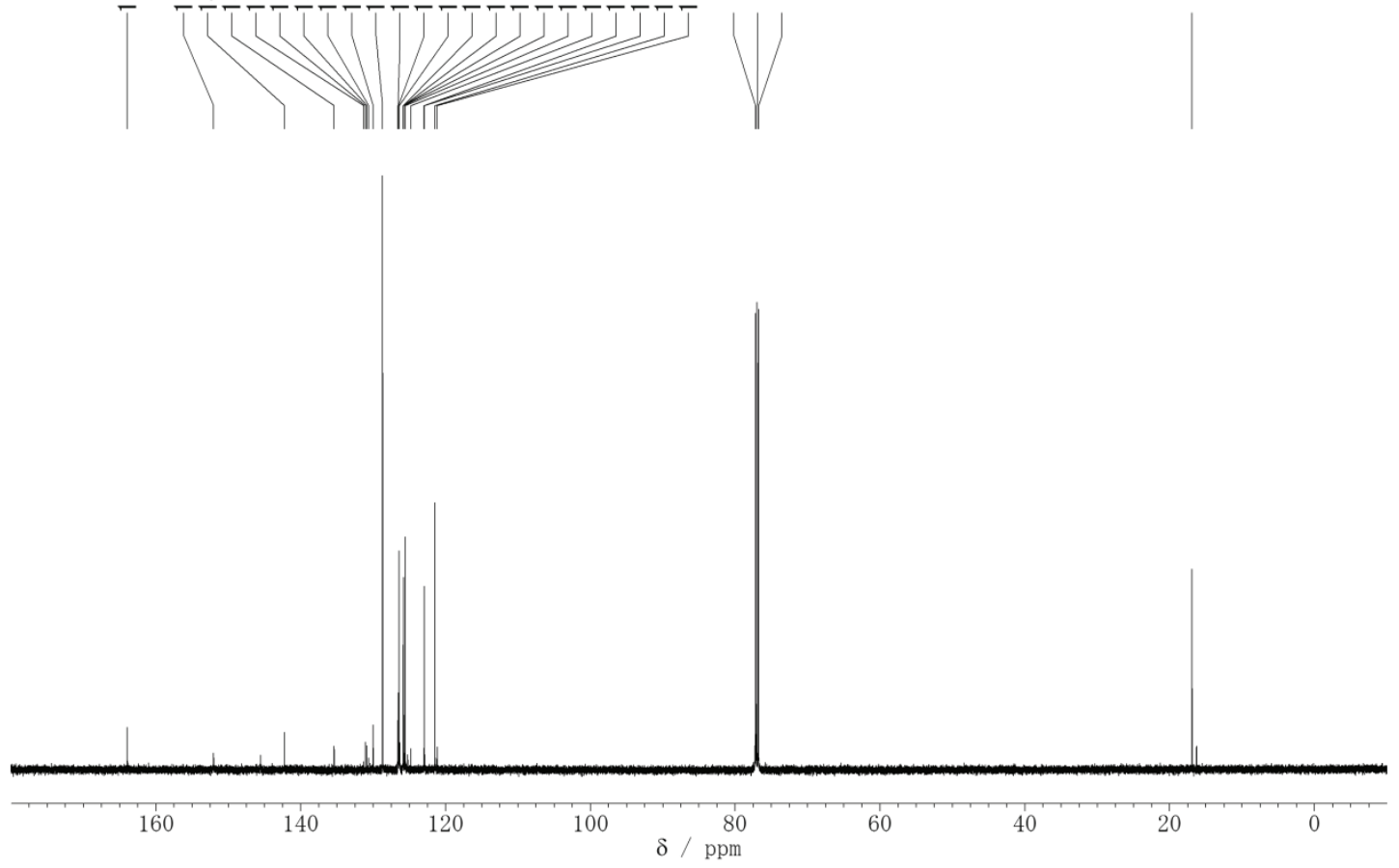


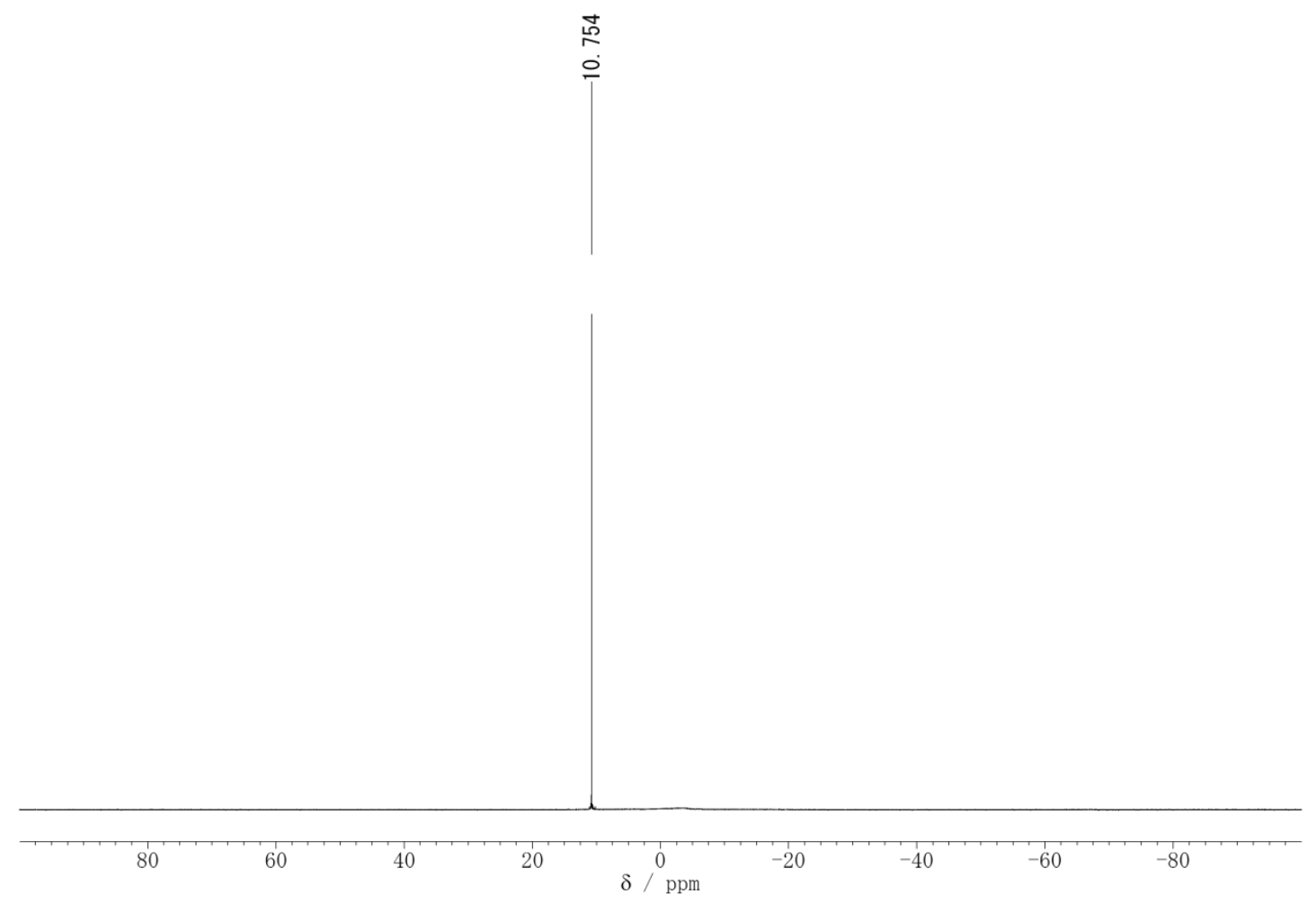

${ }^{1} \mathrm{H}$ NMR (300 MHz), ${ }^{13} \mathrm{C}\left\{{ }^{1} \mathrm{H}\right\}$ NMR (150 MHz), and ${ }^{19} \mathrm{~F}\left\{{ }^{1} \mathrm{H}\right\}$ NMR (300 MHz) spectra of $\mathbf{3 a d}\left(\mathrm{rt}, \mathrm{CDCl}_{3}\right)$. 


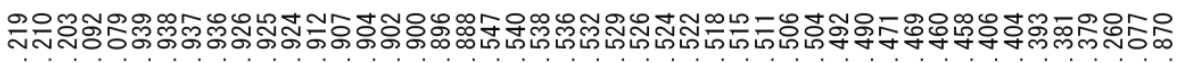
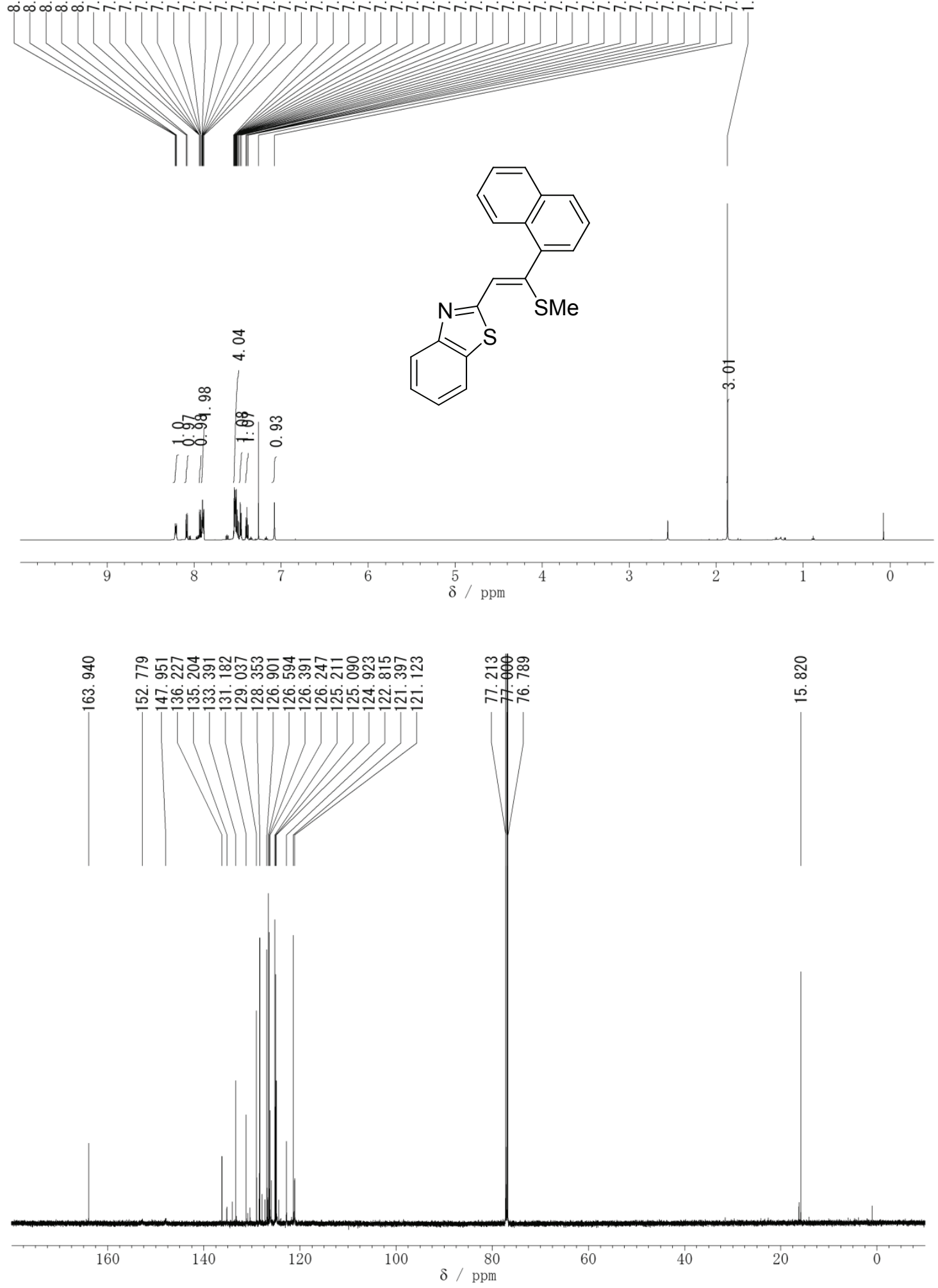

${ }^{1} \mathrm{H}$ NMR $(600 \mathrm{MHz})$ and ${ }^{13} \mathrm{C}\left\{{ }^{1} \mathrm{H}\right\}$ NMR $(150 \mathrm{MHz})$ spectra of $\mathbf{3 a e}\left(\mathrm{rt}, \mathrm{CDCl}_{3}\right)$. 

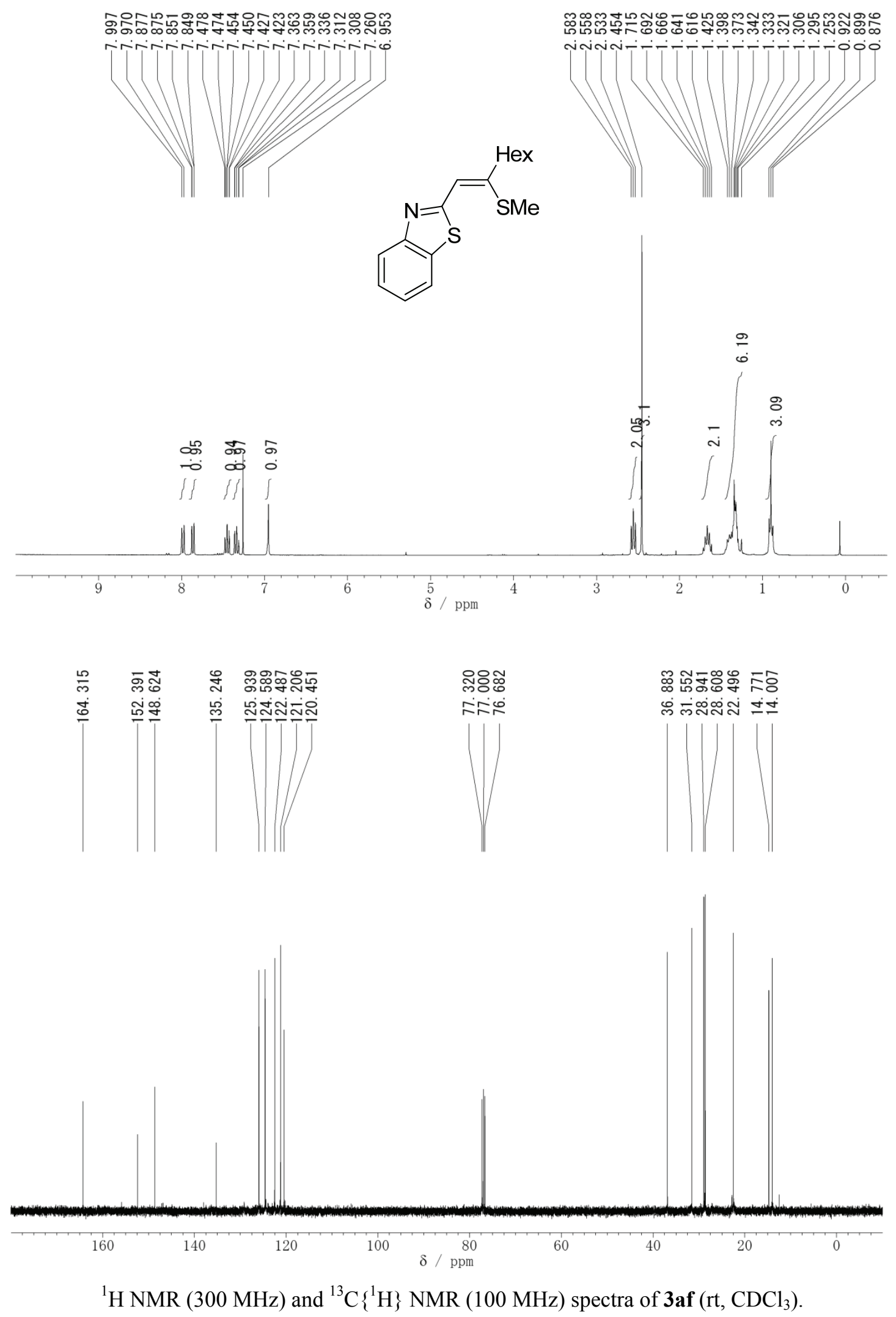

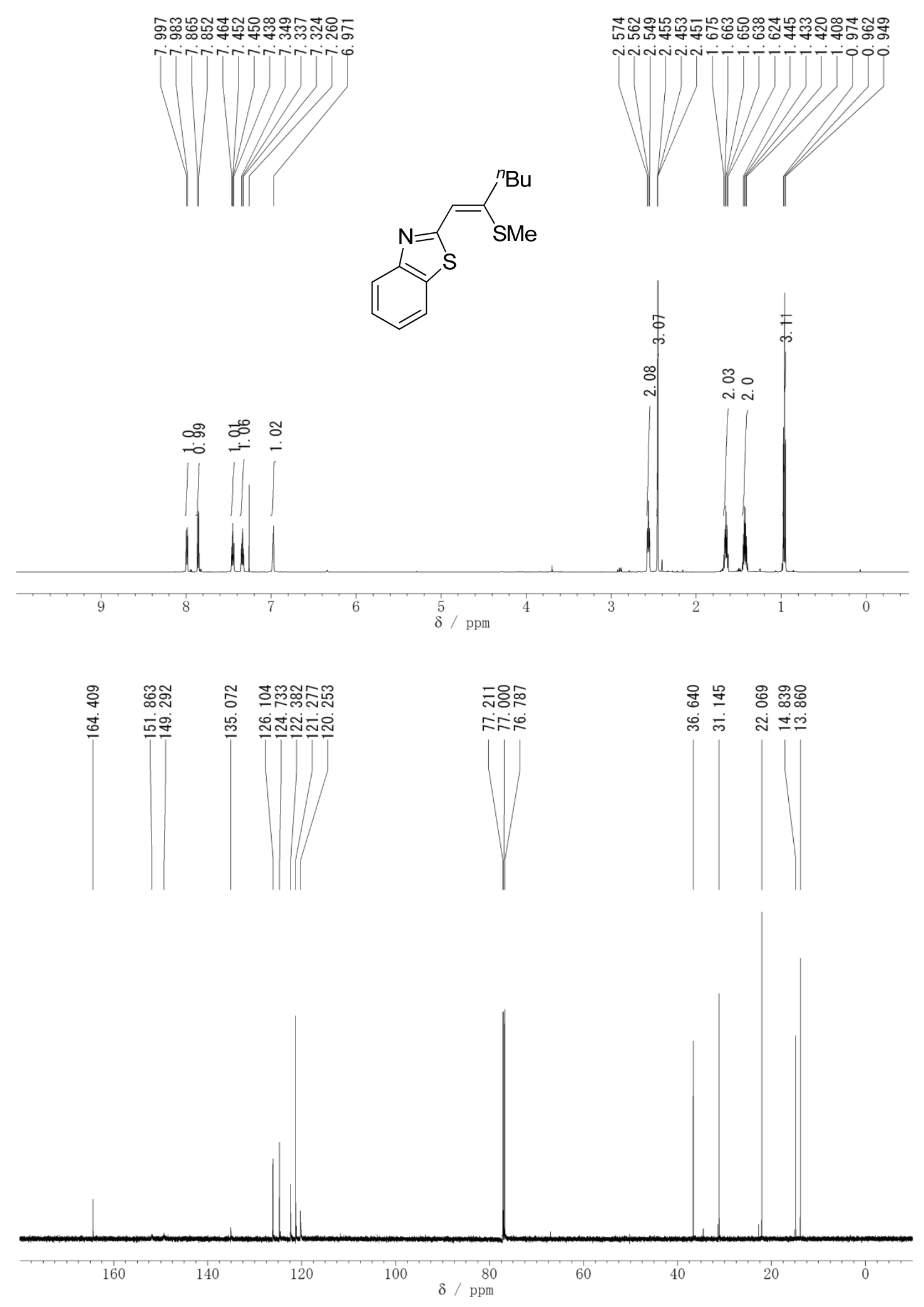

${ }^{1} \mathrm{H}$ NMR $(600 \mathrm{MHz})$ and ${ }^{13} \mathrm{C}\left\{{ }^{1} \mathrm{H}\right\}$ NMR $(150 \mathrm{MHz})$ spectra of $\mathbf{3 a g}\left(\mathrm{rt}, \mathrm{CDCl}_{3}\right)$. 

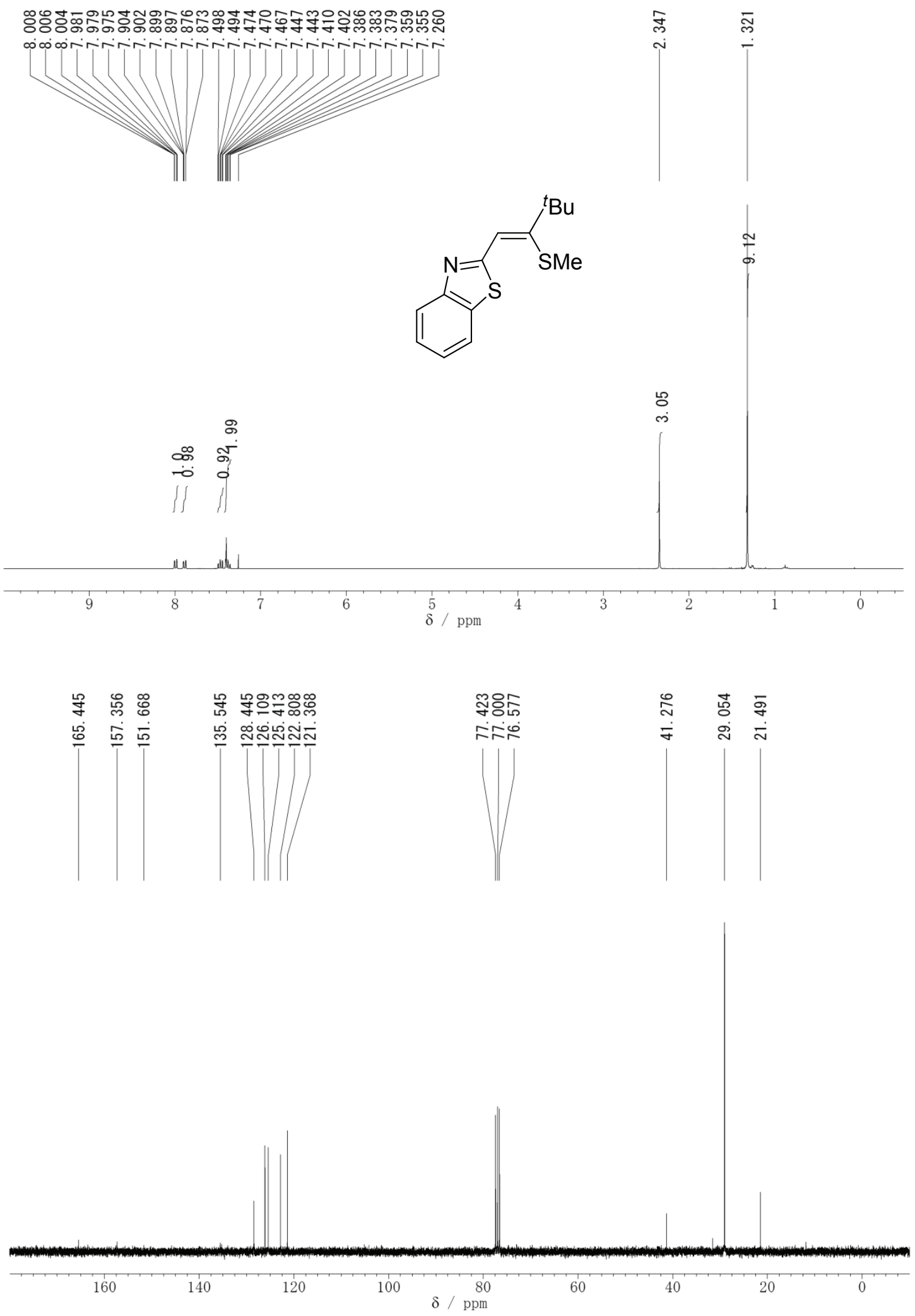

${ }^{1} \mathrm{H}$ NMR (300 MHz) and ${ }^{13} \mathrm{C}\left\{{ }^{1} \mathrm{H}\right\}$ NMR (75 MHz) spectra of 3ah (rt, $\left.\mathrm{CDCl}_{3}\right)$. 

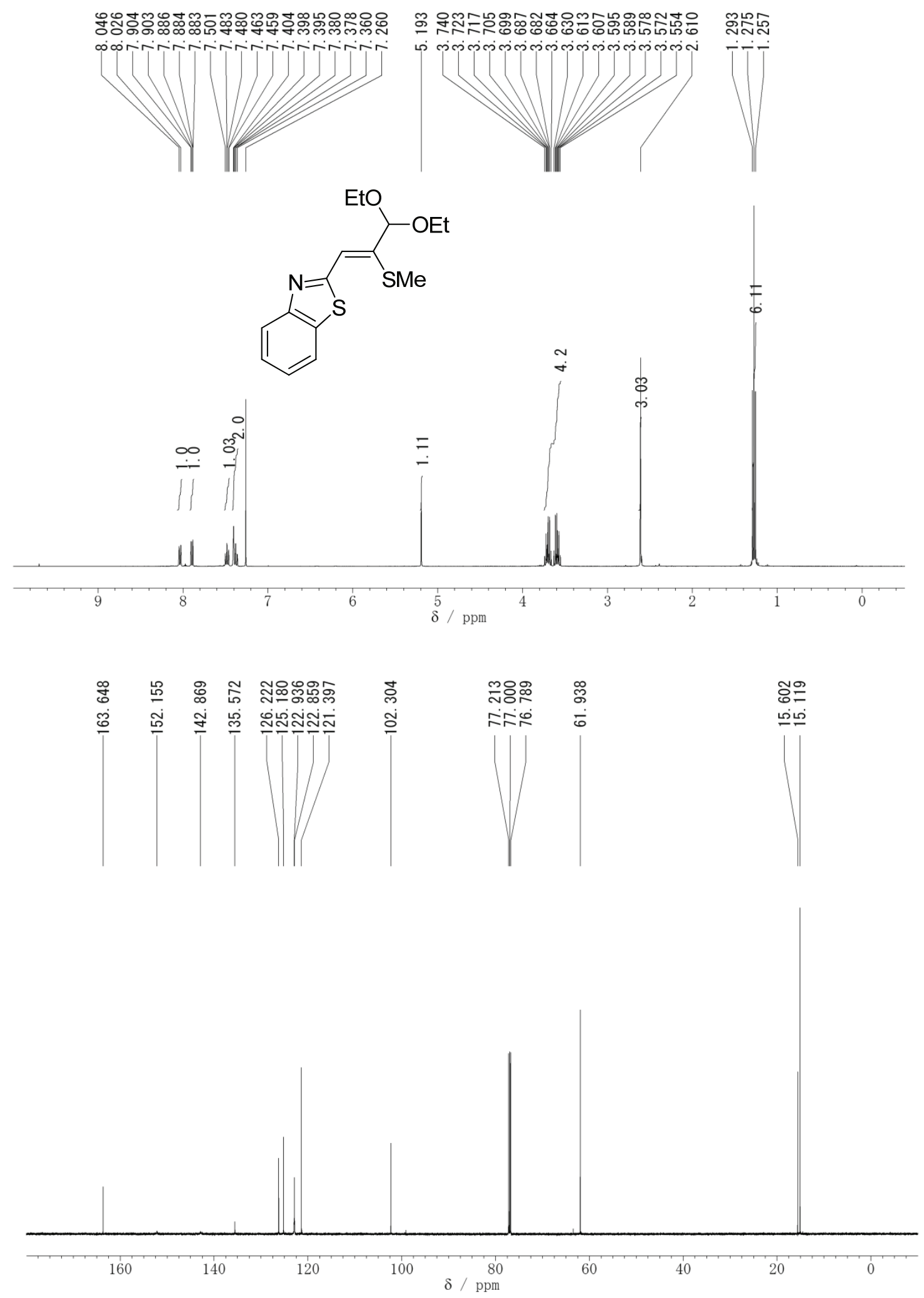

${ }^{1} \mathrm{H}$ NMR (400 MHz) and ${ }^{13} \mathrm{C}\left\{{ }^{1} \mathrm{H}\right\}$ NMR (150 MHz) spectra of 3ai (rt, $\left.\mathrm{CDCl}_{3}\right)$. 

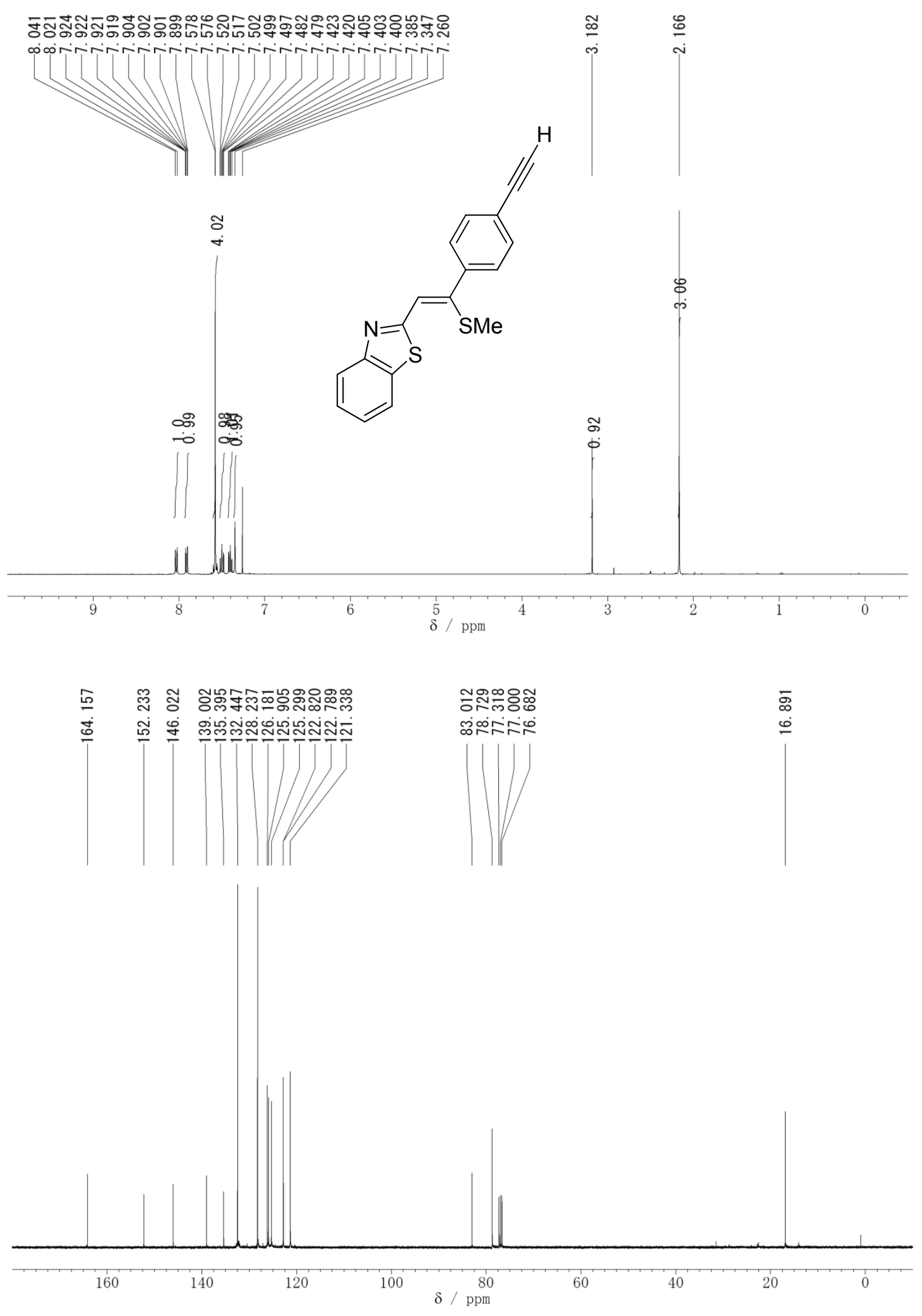

${ }^{1} \mathrm{H}$ NMR $(400 \mathrm{MHz})$ and ${ }^{13} \mathrm{C}\left\{{ }^{1} \mathrm{H}\right\}$ NMR $(100 \mathrm{MHz})$ spectra of 3aj $\left(\mathrm{rt}, \mathrm{CDCl}_{3}\right)$. 

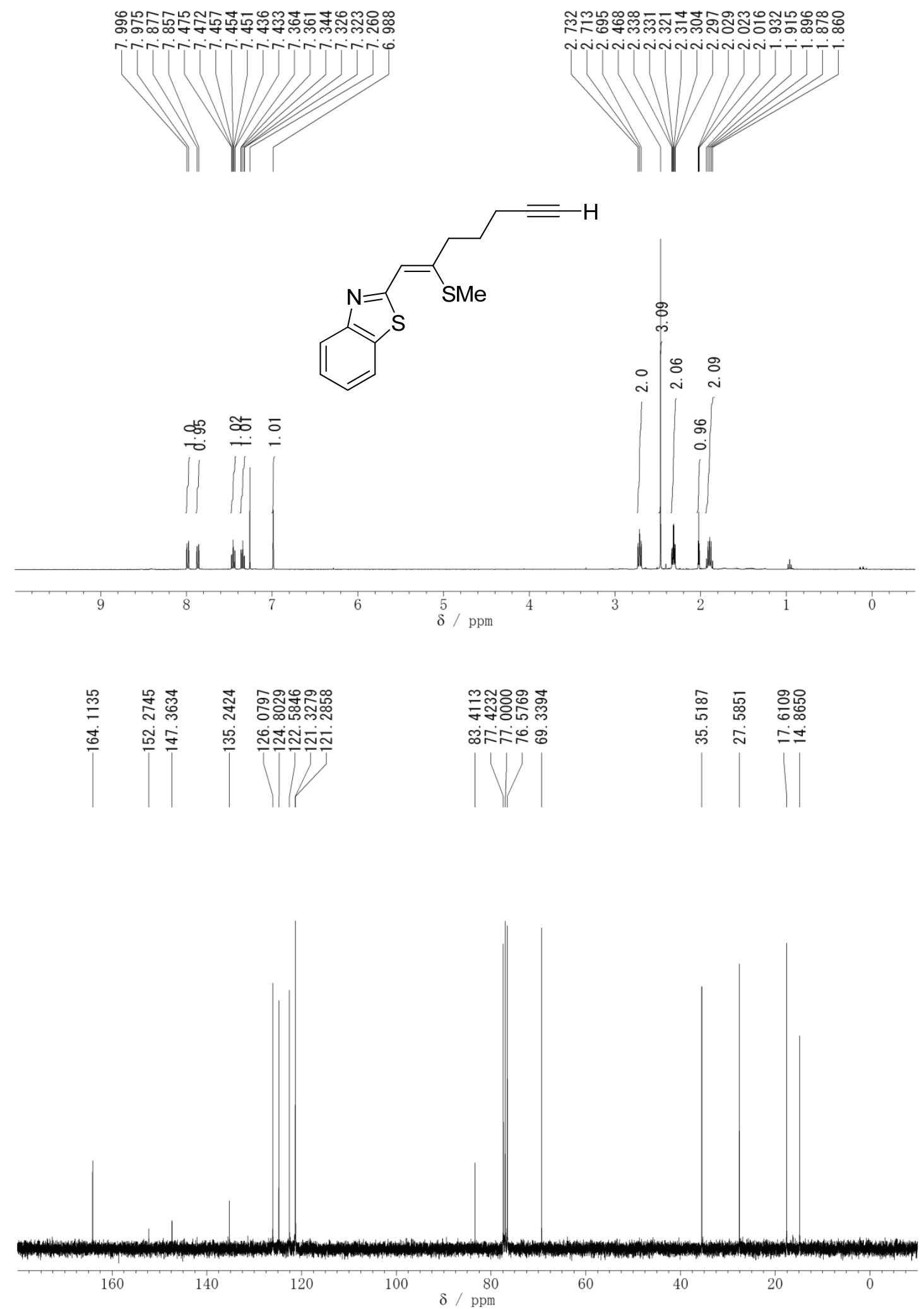

${ }^{1} \mathrm{H}$ NMR $(400 \mathrm{MHz})$ and ${ }^{13} \mathrm{C}\left\{{ }^{1} \mathrm{H}\right\}$ NMR $(75 \mathrm{MHz})$ spectra of 3ak (rt, $\left.\mathrm{CDCl}_{3}\right)$. 

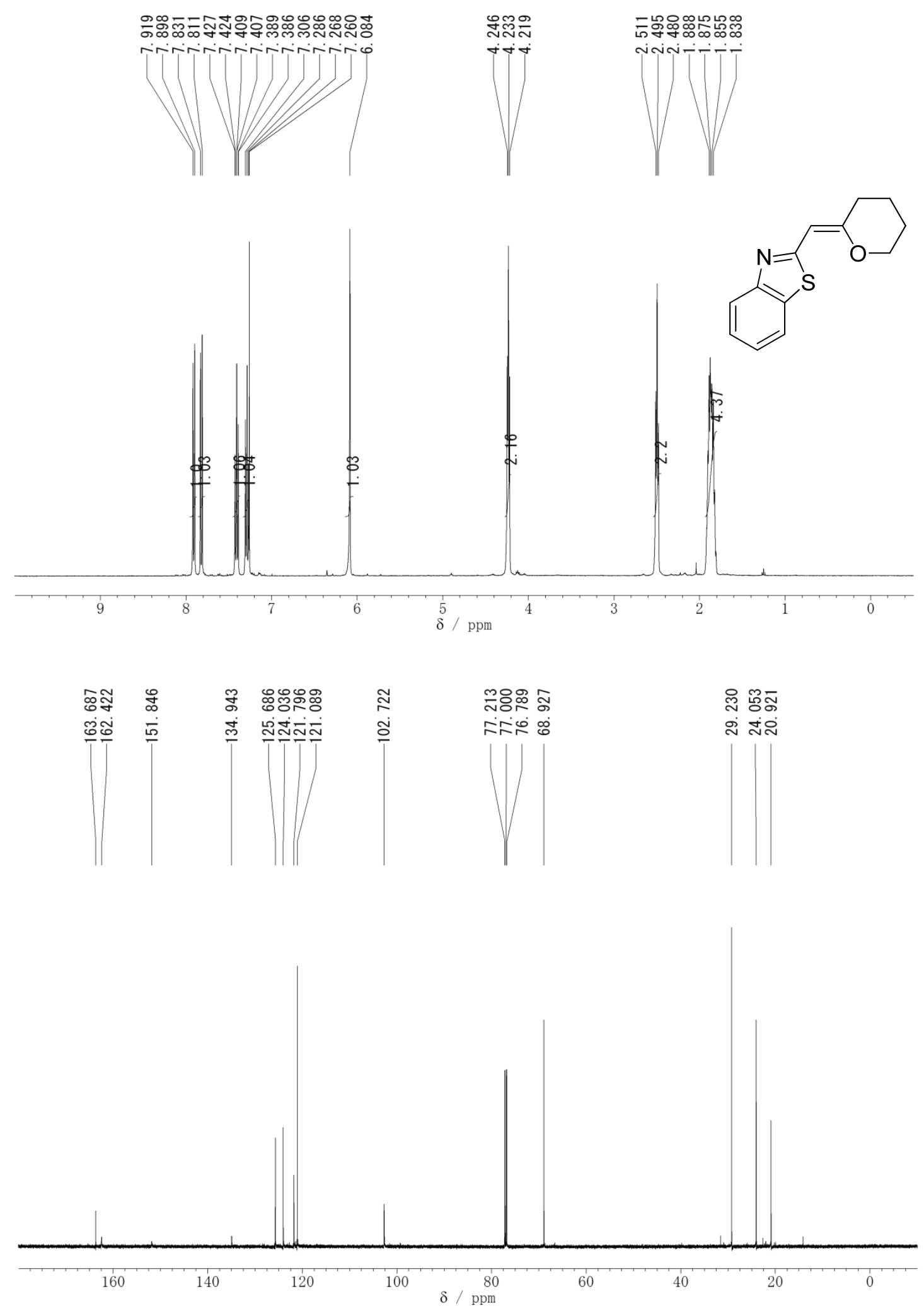

${ }^{1} \mathrm{H}$ NMR (400 MHz) and ${ }^{13} \mathrm{C}\left\{{ }^{1} \mathrm{H}\right\}$ NMR (150 MHz) spectra of 3al' (rt, $\left.\mathrm{CDCl}_{3}\right)$. 

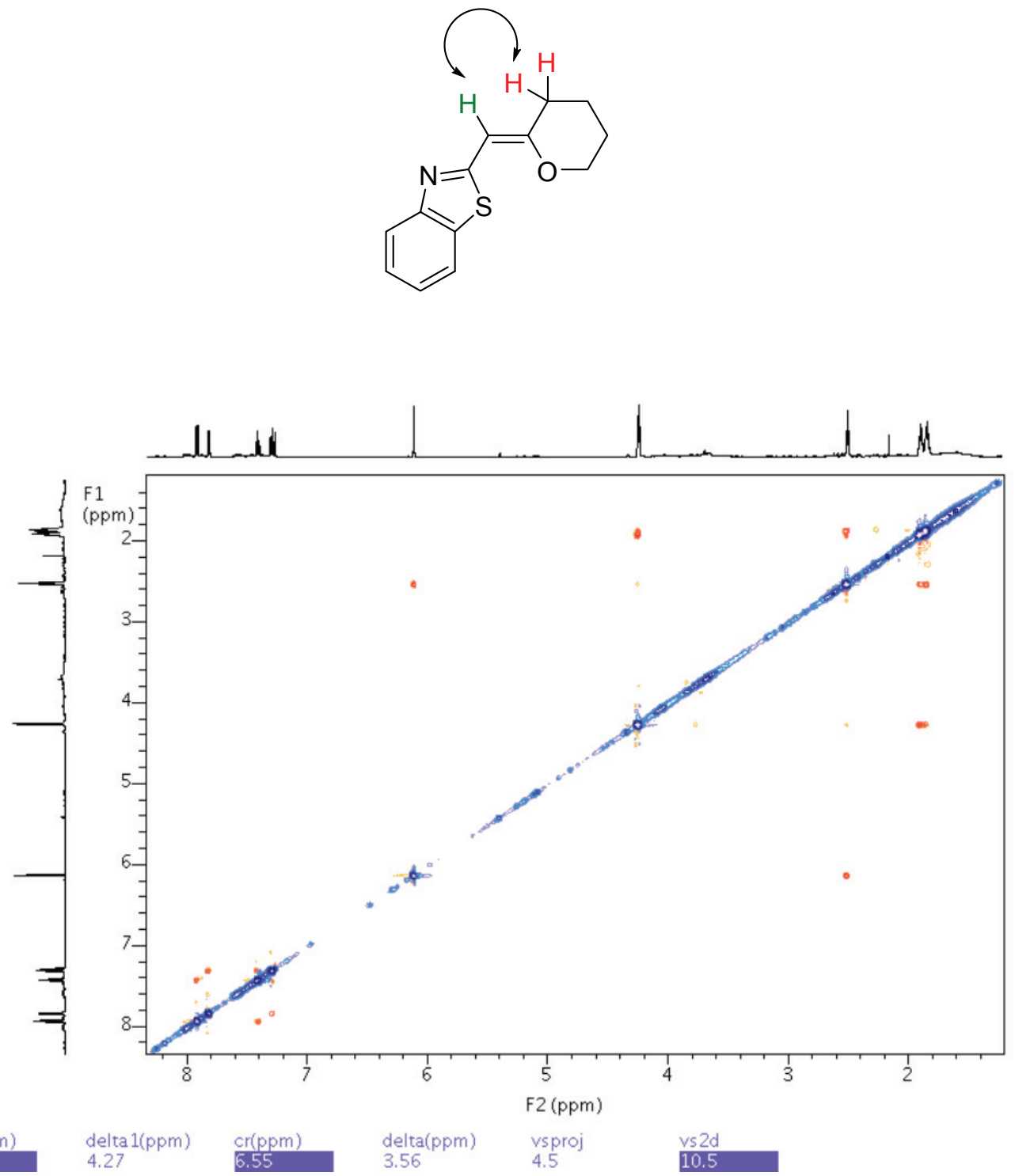

NOESY (600 MHz) spectrum of 3al' ( $\left(\mathrm{rt}, \mathrm{CDCl}_{3}\right.$ ) clearly showing the correlation between the allylic methylene protons and the vinyl proton $(\delta=2.49 \mathrm{ppm}$ and $6.08 \mathrm{ppm})$. 

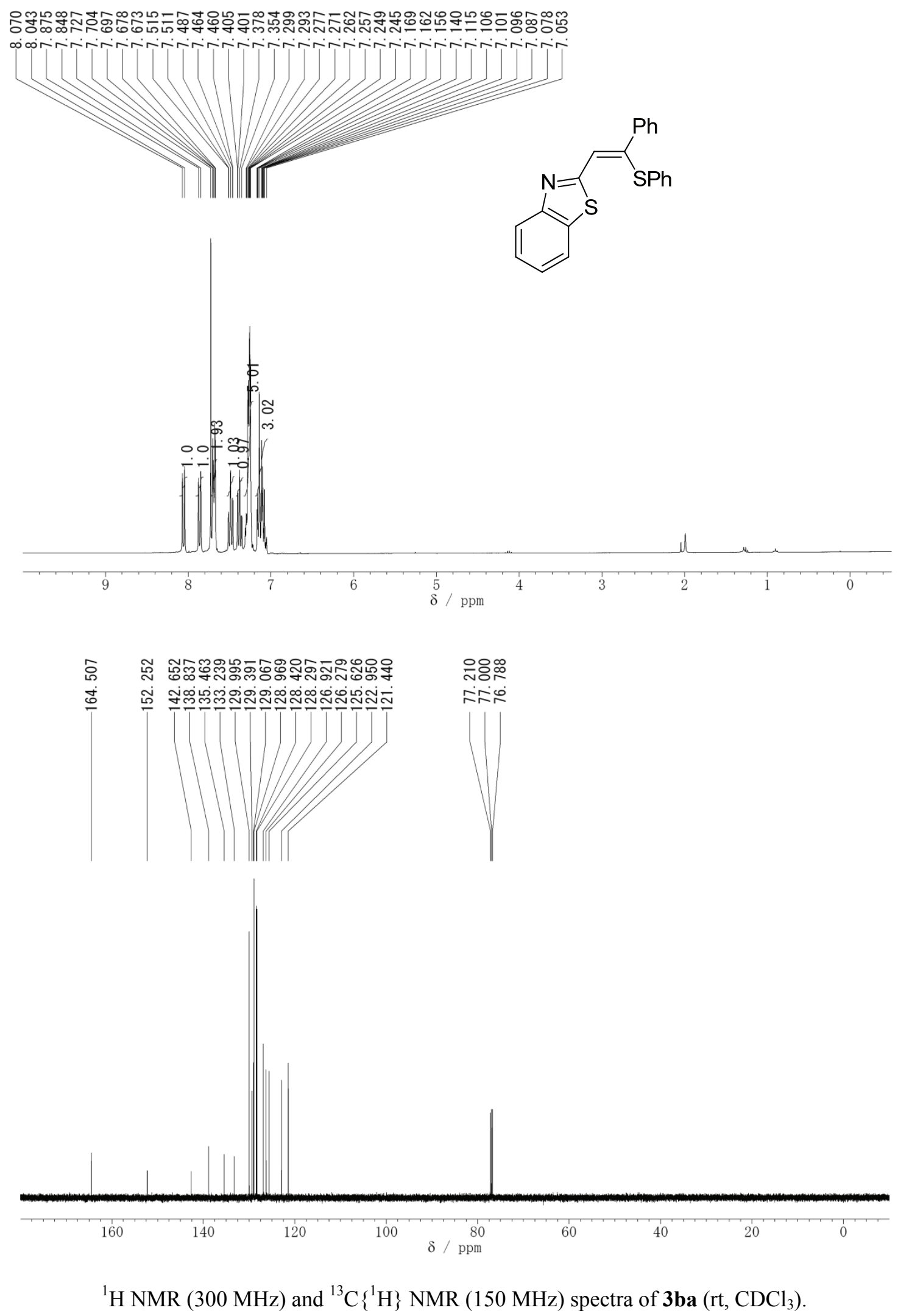

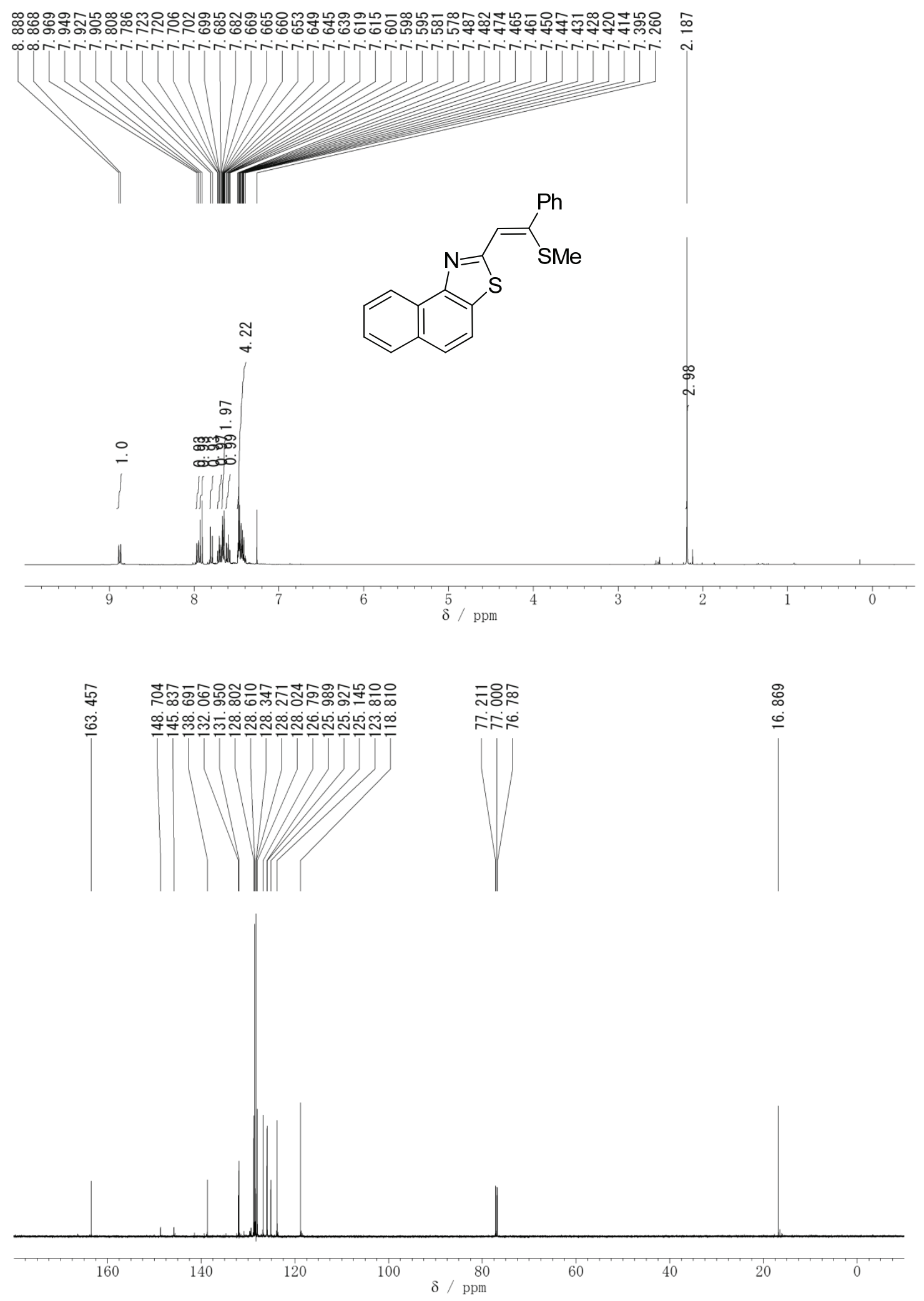

${ }^{1} \mathrm{H}$ NMR $(400 \mathrm{MHz})$ and ${ }^{13} \mathrm{C}\left\{{ }^{1} \mathrm{H}\right\}$ NMR $(150 \mathrm{MHz})$ spectra of $\mathbf{3} \mathbf{c a}\left(\mathrm{rt}, \mathrm{CDCl}_{3}\right)$. 

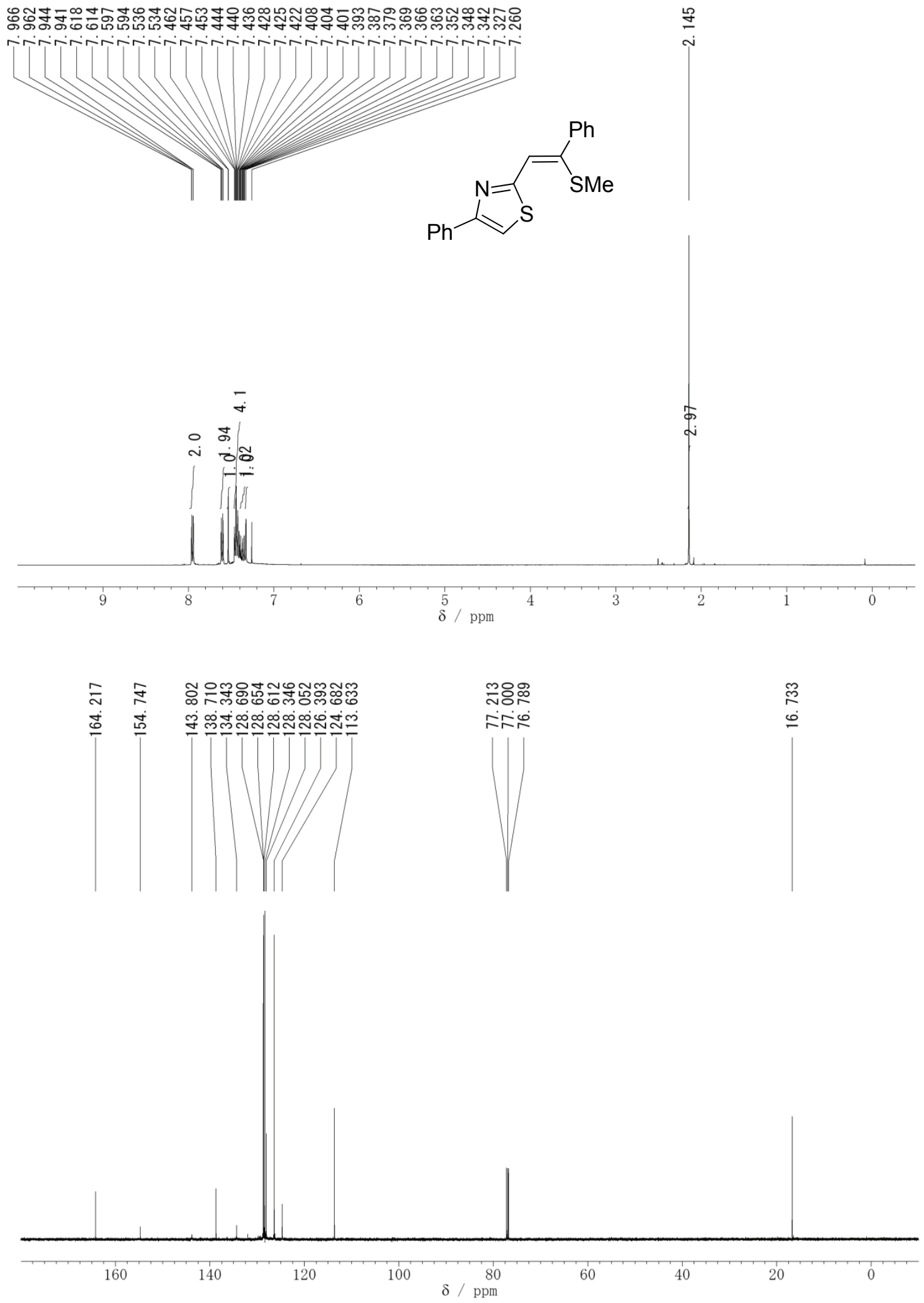

${ }^{1} \mathrm{H}$ NMR $(600 \mathrm{MHz})$ and ${ }^{13} \mathrm{C}\left\{{ }^{1} \mathrm{H}\right\}$ NMR $(150 \mathrm{MHz})$ spectra of 3da $\left(\mathrm{rt}, \mathrm{CDCl}_{3}\right)$. 

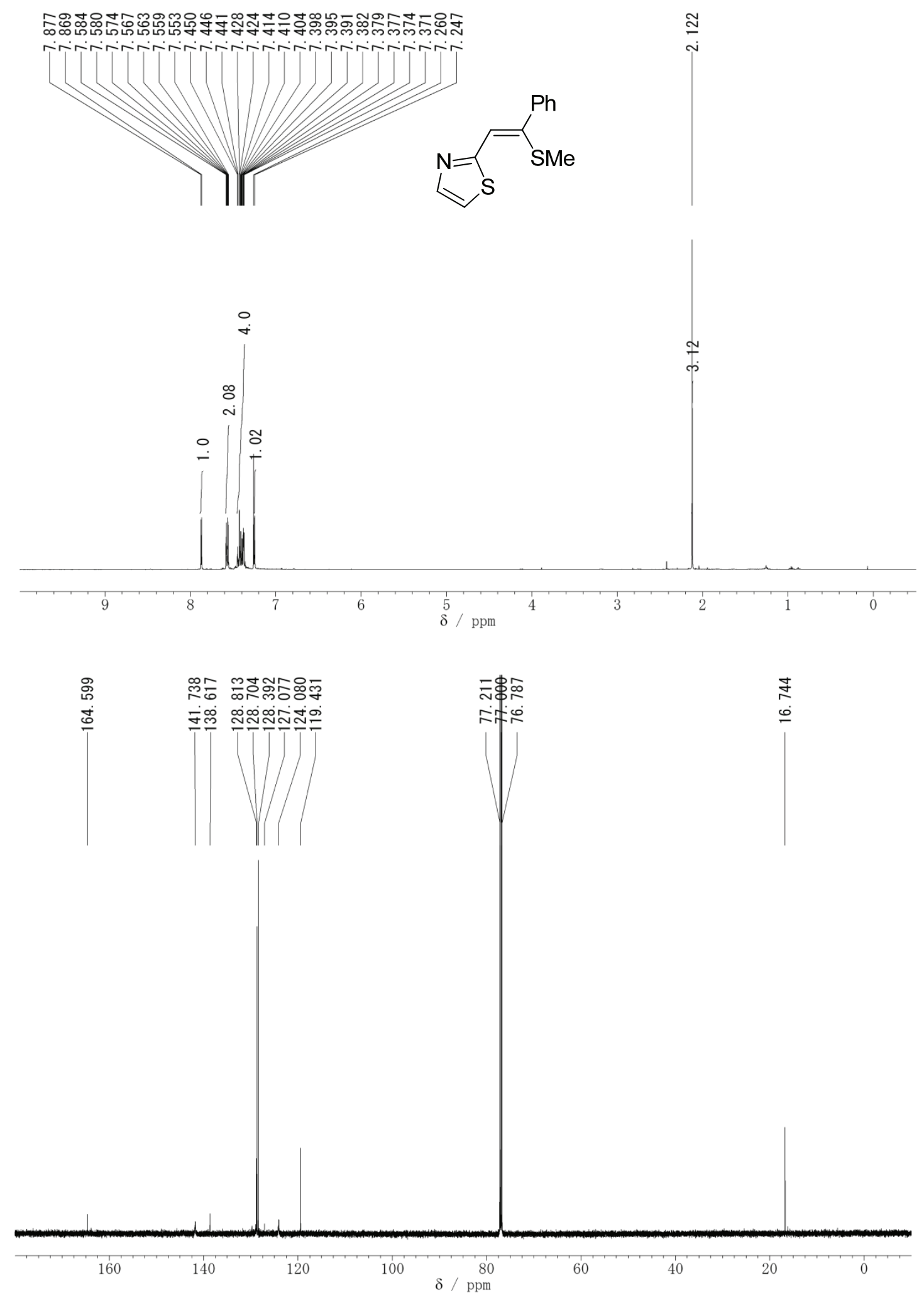

${ }^{1} \mathrm{H}$ NMR $(400 \mathrm{MHz})$ and ${ }^{13} \mathrm{C}\left\{{ }^{1} \mathrm{H}\right\}$ NMR $(150 \mathrm{MHz})$ spectra of 3ea (rt, $\left.\mathrm{CDCl}_{3}\right)$. 

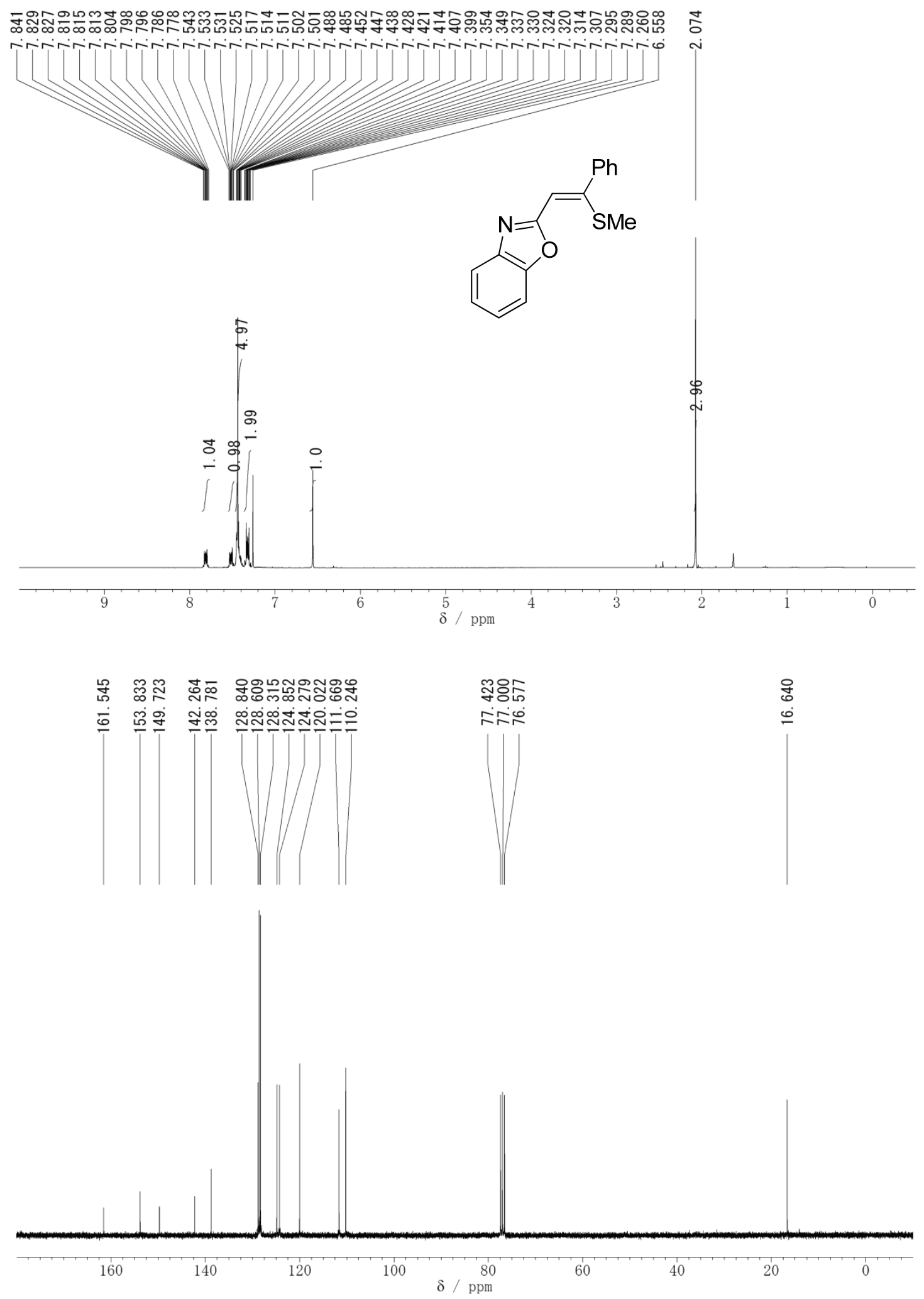

${ }^{1} \mathrm{H}$ NMR $(300 \mathrm{MHz})$ and ${ }^{13} \mathrm{C}\left\{{ }^{1} \mathrm{H}\right\}$ NMR $(75 \mathrm{MHz})$ spectra of $\mathbf{3 f a}\left(\mathrm{rt}, \mathrm{CDCl}_{3}\right)$. 

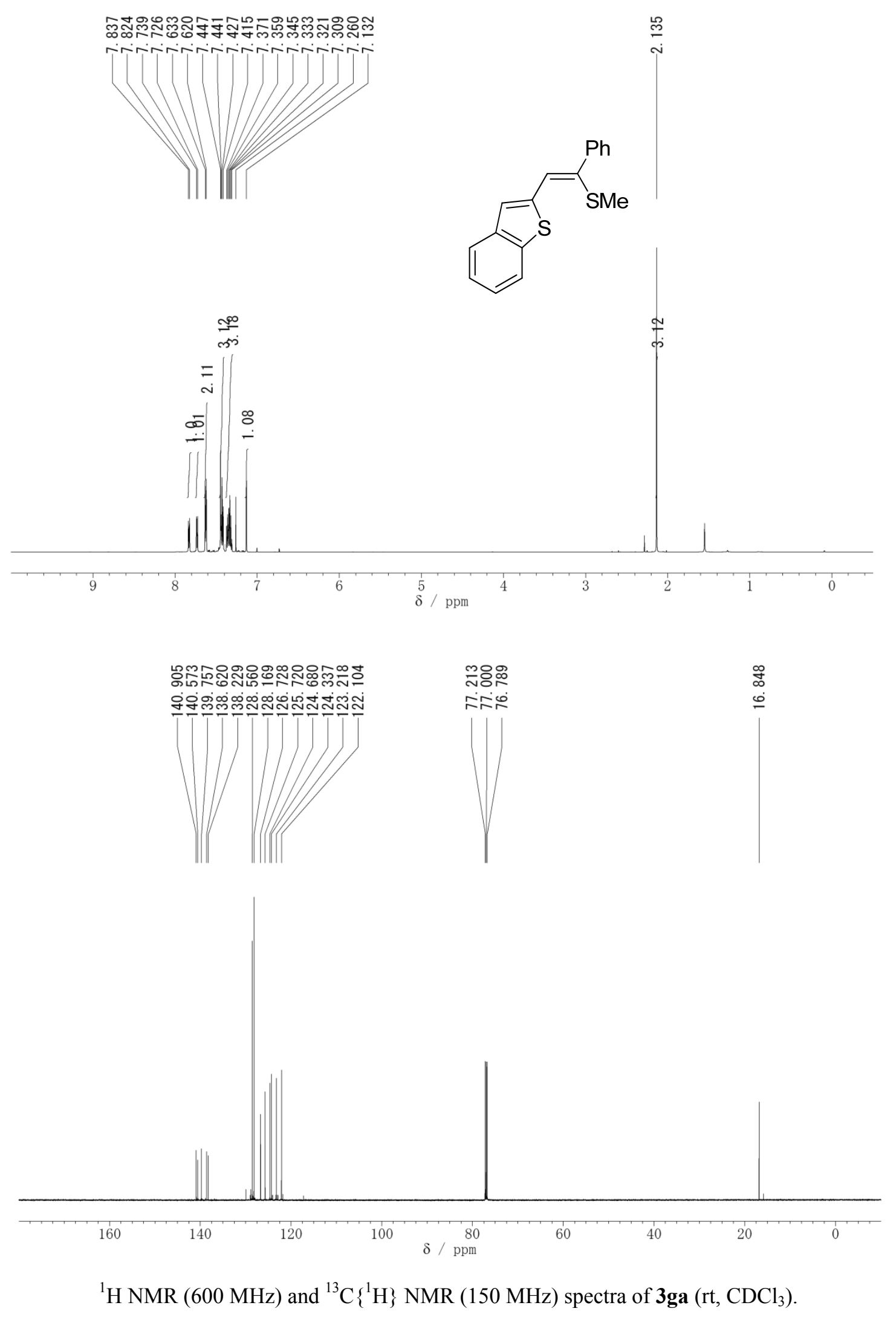


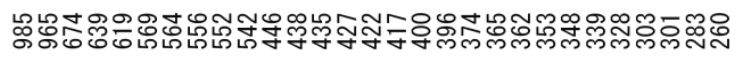
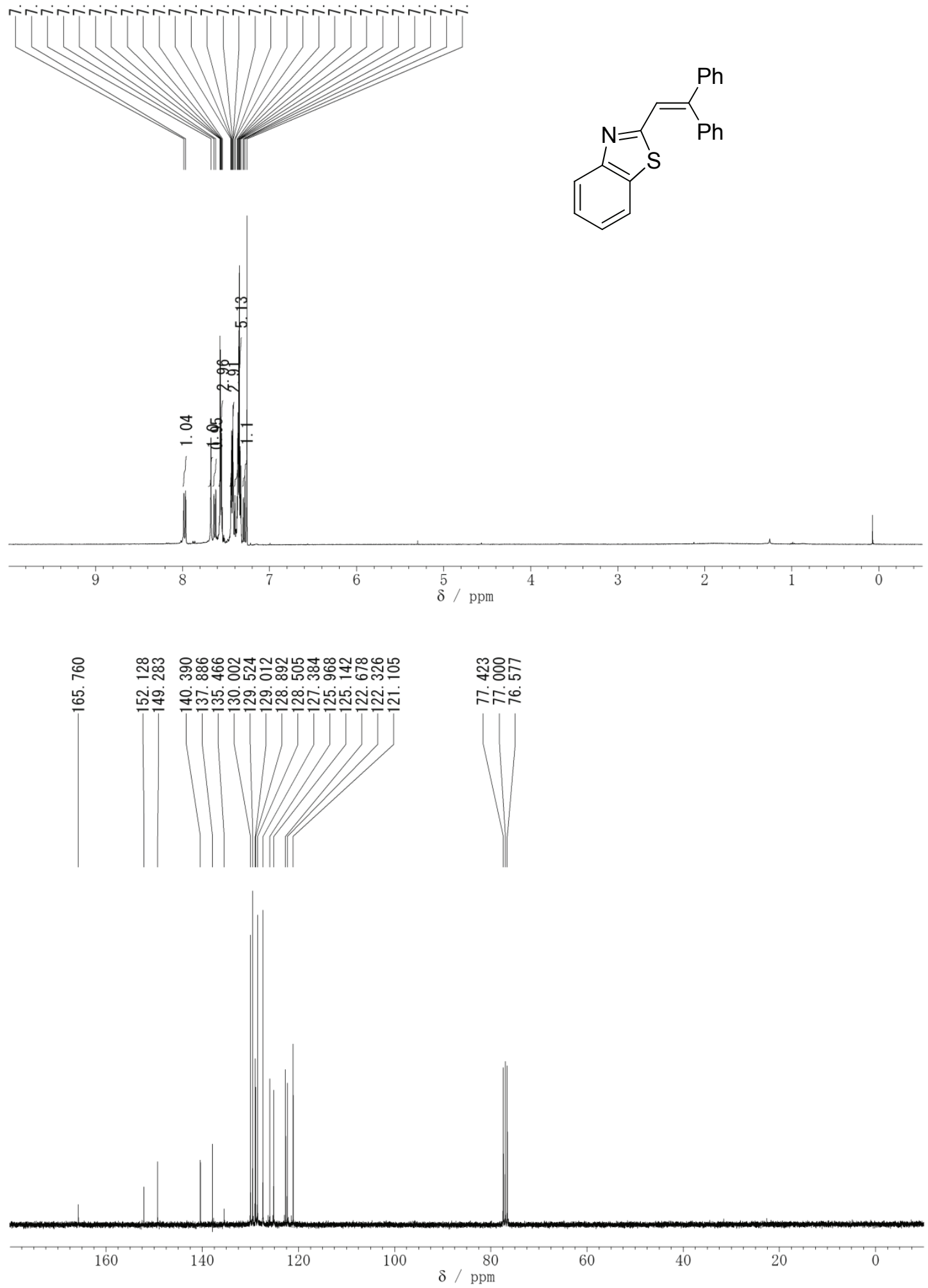

${ }^{1} \mathrm{H}$ NMR (400 MHz) and ${ }^{13} \mathrm{C}\left\{{ }^{1} \mathrm{H}\right\}$ NMR (75 MHz) spectra of $4\left(\mathrm{rt}, \mathrm{CDCl}_{3}\right)$. 


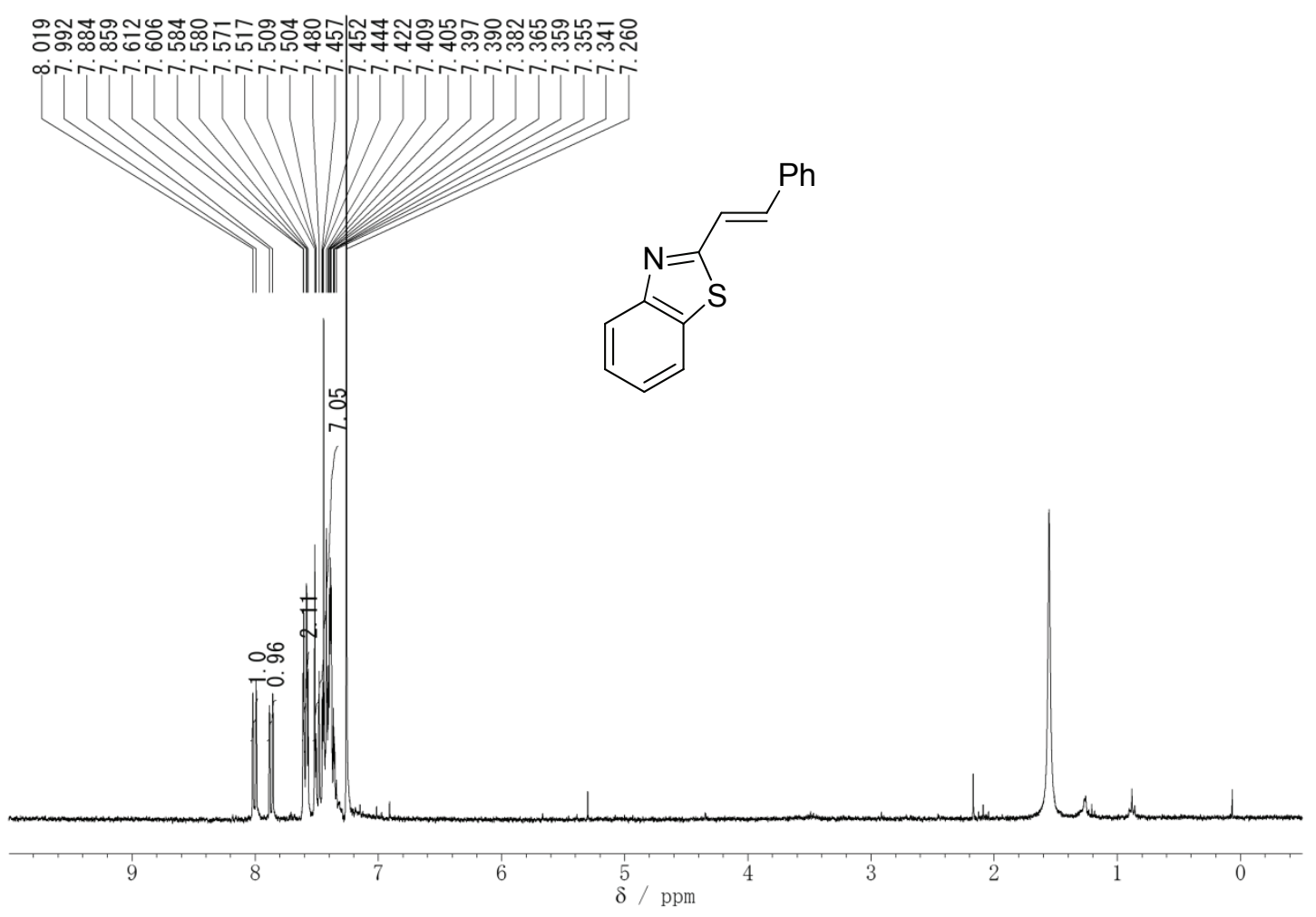

${ }^{1} \mathrm{H}$ NMR (300 MHz) spectrum of $5\left(\mathrm{rt}, \mathrm{CDCl}_{3}\right)$. 

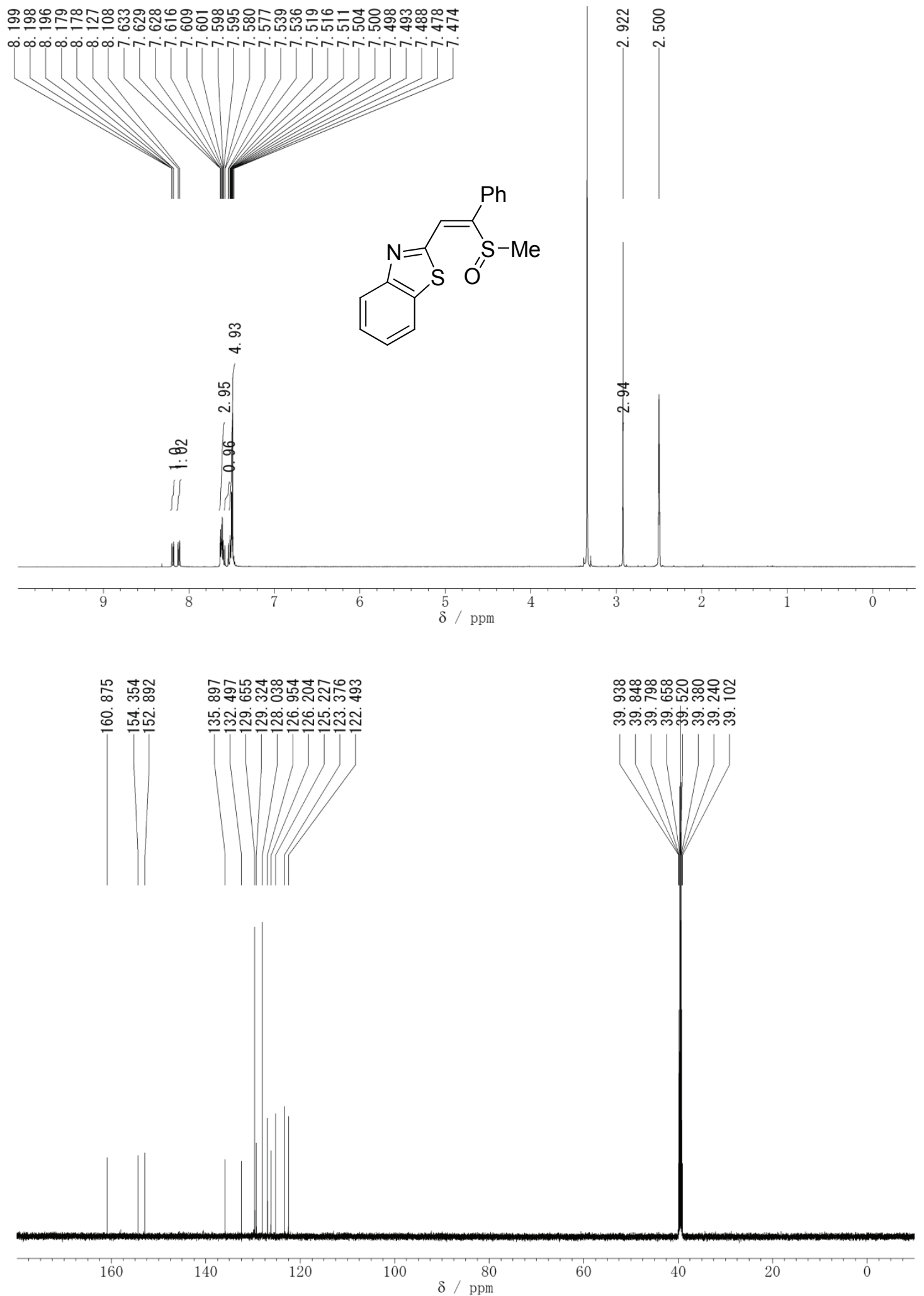

${ }^{1} \mathrm{H}$ NMR (400 MHz) and ${ }^{13} \mathrm{C}\left\{{ }^{1} \mathrm{H}\right\}$ NMR (150 MHz) spectra of 6 (rt, DMSO- $d_{6}$ ). 

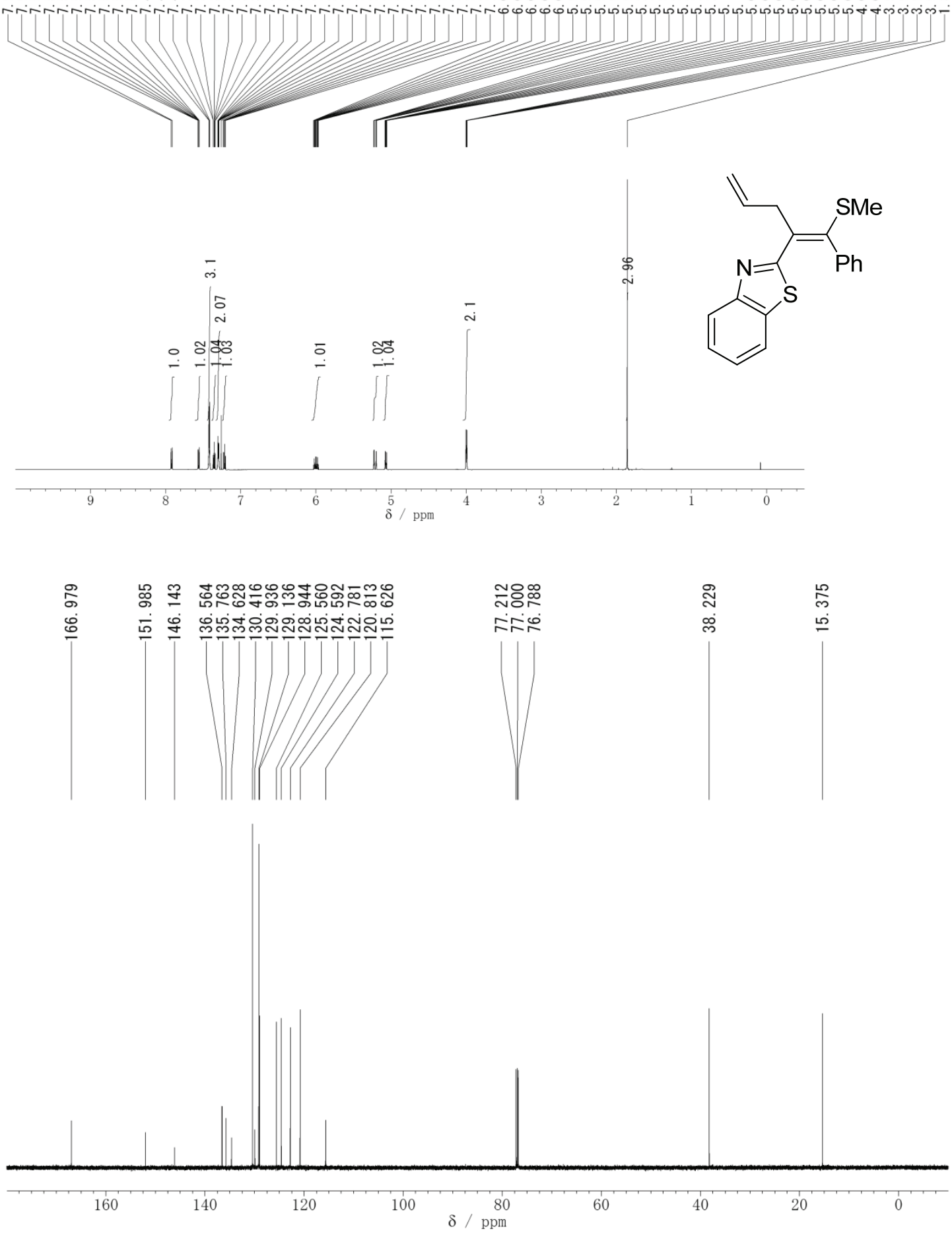

${ }^{1} \mathrm{H}$ NMR $(600 \mathrm{MHz})$ and ${ }^{13} \mathrm{C}\left\{{ }^{1} \mathrm{H}\right\}$ NMR (150 MHz) spectra of $(E)-7$ (rt, $\left.\mathrm{CDCl}_{3}\right)$. 


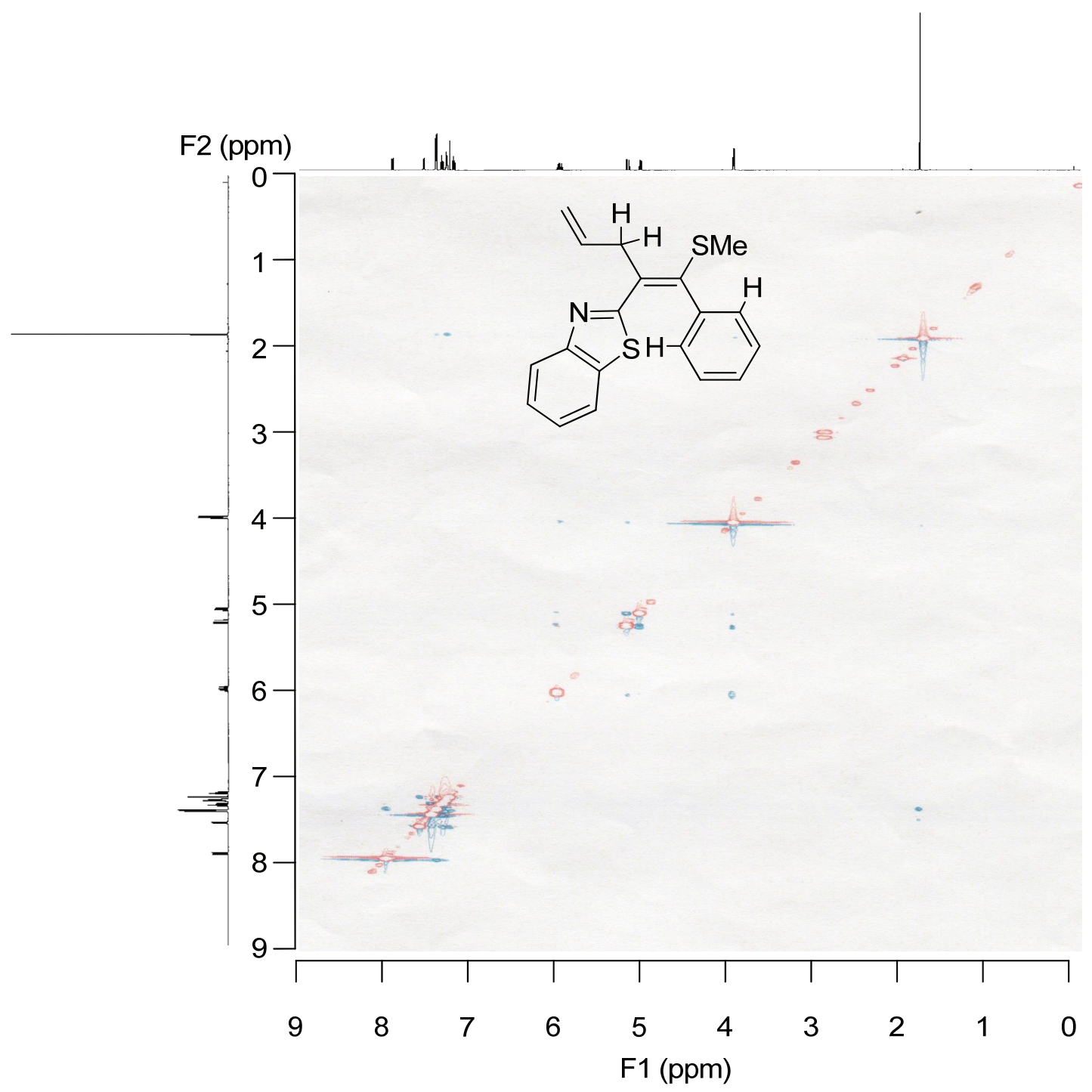

NOESY (600 MHz) spectrum of $(E)-7$ (rt, $\left.\mathrm{CDCl}_{3}\right)$ clearly showing no correlation between the allylic methylene protons and the aryl protons ( $\delta=4.00 \mathrm{ppm}$ and $7.29-7.31 \mathrm{ppm})$. 

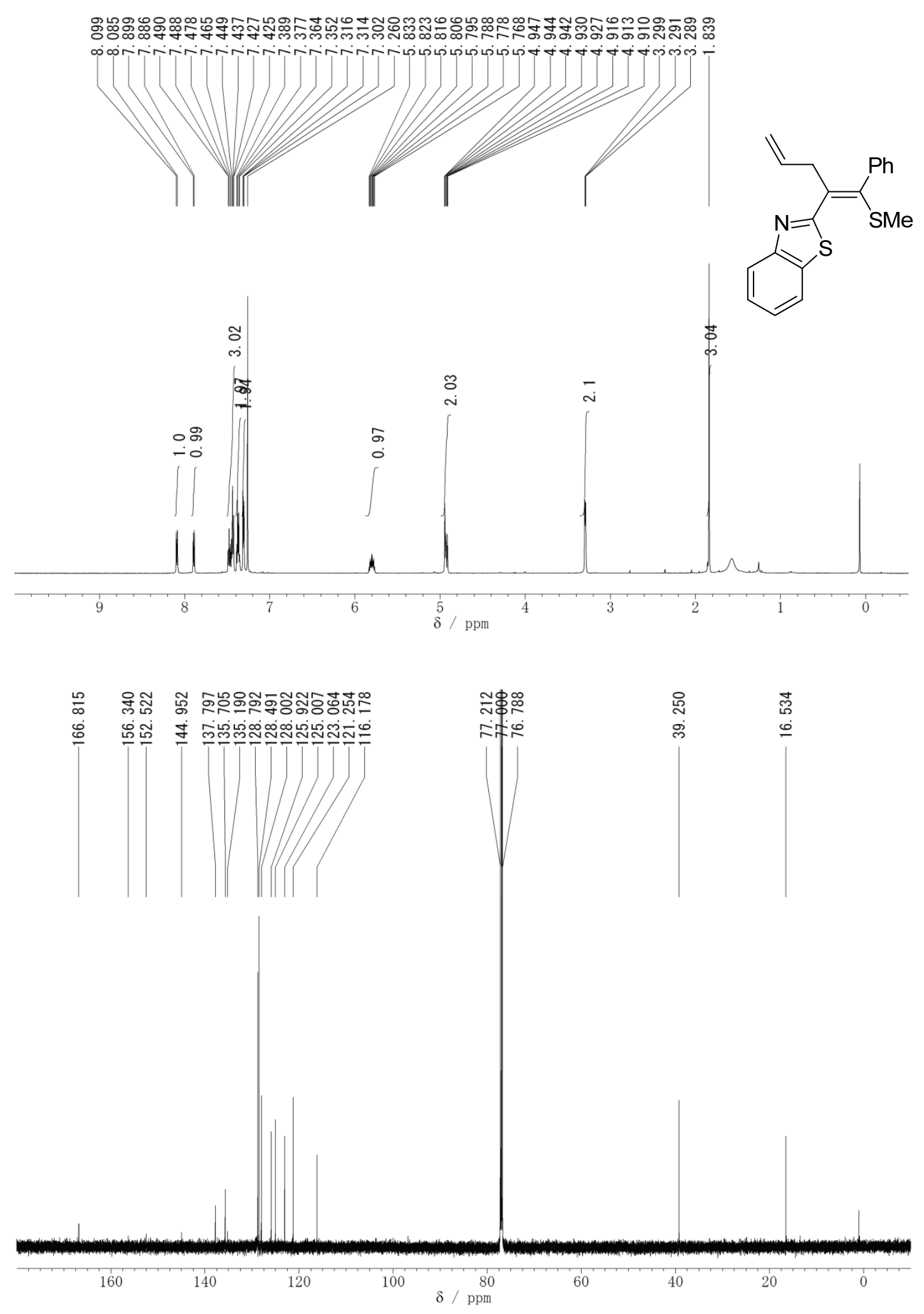

${ }^{1} \mathrm{H}$ NMR (600 MHz) and ${ }^{13} \mathrm{C}\left\{{ }^{1} \mathrm{H}\right\}$ NMR (150 MHz) spectra of (Z)-7 (rt, $\left.\mathrm{CDCl}_{3}\right)$. 


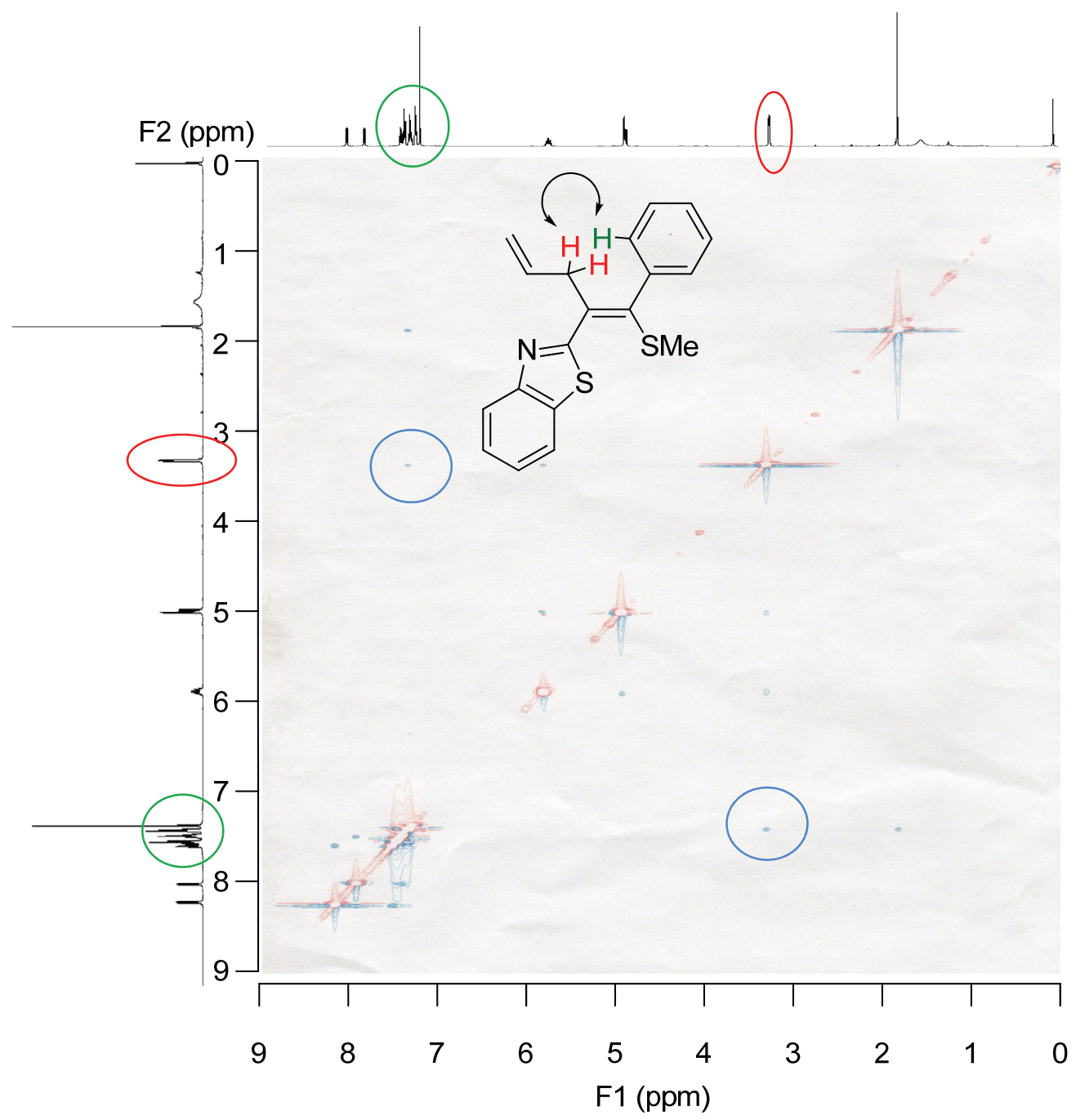

NOESY $(600 \mathrm{MHz})$ spectrum of $(Z)-7\left(\mathrm{rt}, \mathrm{CDCl}_{3}\right)$ clearly showing the correlation between the allylic methylene protons and the aryl protons ( $\delta=3.30 \mathrm{ppm}$ and $7.35-7.39 \mathrm{ppm})$. 


\section{A Plausible Reaction Mechanism of Allylation of Sulfoxide 6}

The regio- and stereoselectivities of allylation would be rationalized as shown in Scheme S1. First, sulfonium salt $\mathbf{C}$ is generated by the reaction of sulfoxide $\mathbf{6}$ with trifluoromethanesulfonic anhydride $\left(\mathrm{Tf}_{2} \mathrm{O}\right) .{ }^{13}$ The subsequent nucleophilic attack of allylsilane on the cationic sulfur of $\mathbf{D}$ gives sulfonium salt $\mathbf{E}$ with a release of trimethylsilyl triflate. ${ }^{12,14}$ Thio-Claisen rearrangement from $\mathbf{E}$ proceeds via a six-membered chair-like transition state, followed by deprotonation, affording the allylated product 7. ${ }^{15}$ During the stereodetermined deprotonation step, carbon-hydrogen bond should be placed at the perpendicular position of carbon-sulfur double bond from a stereoelectronic viewpoint. There are two possible conformations $\mathbf{E}$ and $\mathbf{E}$ ' in the present reaction. The steric repulsion between the phenyl and allyl groups in $\mathbf{E}$ would retard the following deprotonation, while that between the phenyl and benzothiaolyl groups would be relatively smaller. The preferential deprotonation from $\mathbf{E}$ ' provides $(E)-7$ selectively.

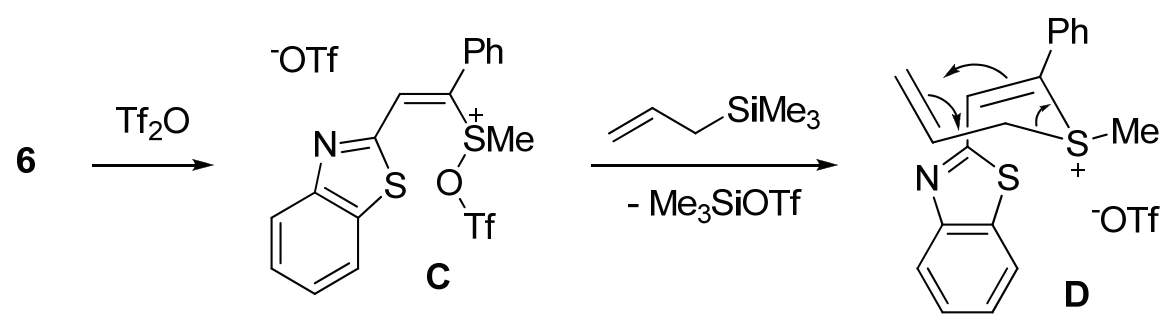

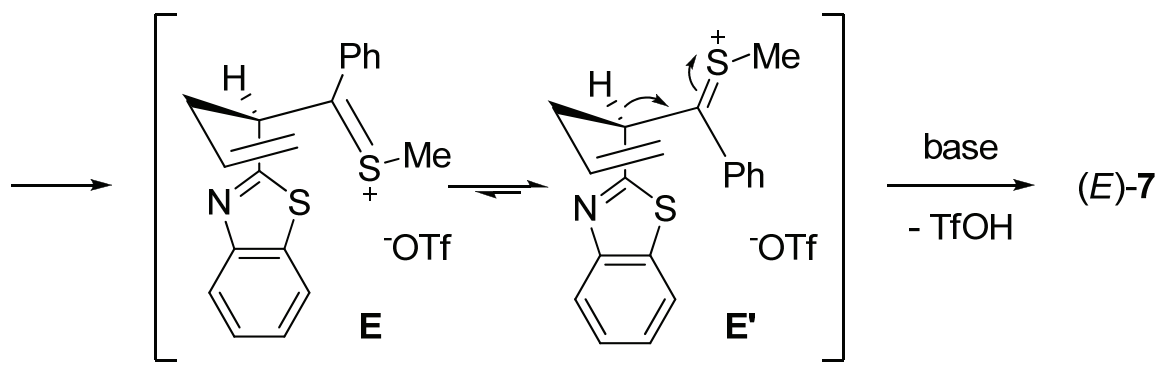<smiles>C=CCC1CC2CC(c3nc4ccccc4s3)(CC2c2ccccc2)C1CC=C</smiles>

E

(disfavored)

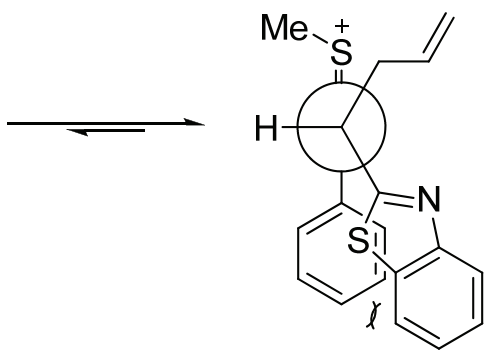

$E^{\prime}$ (favored)

Scheme S1. A plausible reaction mechanism of allylation. 


\section{Structural Determination of Compounds by X-ray Analysis}

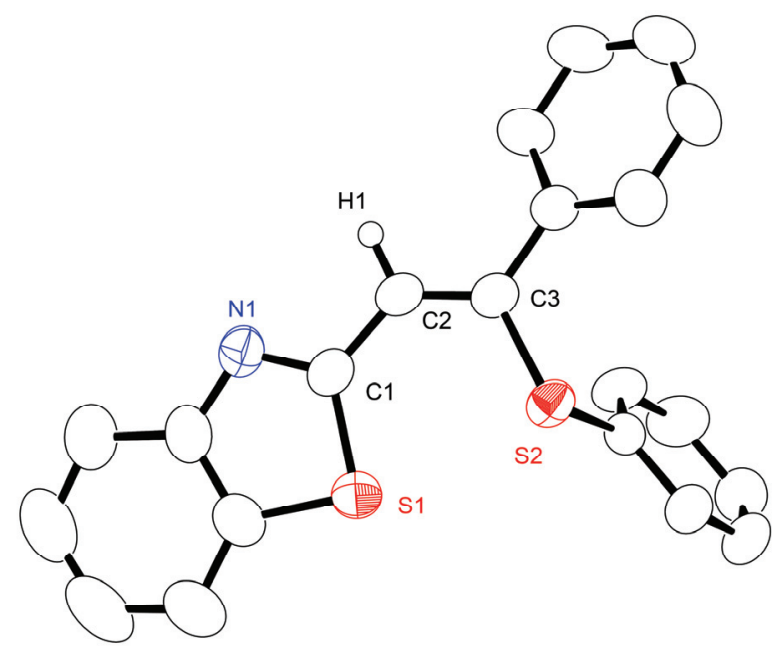

Figure S1. An ORTEP Drawing of $\mathbf{3 b a}$.

\section{A. Crystal Data}

Empirical Formula

$\mathrm{C}_{21} \mathrm{H}_{15} \mathrm{NS}_{2}$

Formula Weight

345.48

Crystal Color, Habit

colorless, prism

Crystal Dimensions

$0.50 \times 0.20 \times 0.20 \mathrm{~mm}$

Crystal System

triclinic

Lattice Type

Primitive

No. of Reflections Used for Unit

Cell Determination ( $2 \theta$ range)

$7878\left(6.4-55.0^{\circ}\right)$

Lattice Parameters

$\mathrm{a}=9.1755(6) \AA$

$\mathrm{b}=10.6216(6) \AA$

$\mathrm{c}=10.7652(6) \AA$

$\mathrm{V}=865.79(9) \AA^{3}$

Space Group

P-1 (\#2)

$Z$ value

2

$\mathrm{D}_{\text {calc }}$

$1.325 \mathrm{~g} / \mathrm{cm}^{3}$

$\mathrm{F}_{000}$

360.00

$\mu(\operatorname{MoK} \alpha)$

$3.081 \mathrm{~cm}^{-1}$

\section{B. Intensity Measurements}

Diffractometer

AFC7

Radiation

$\operatorname{MoK} \alpha(\lambda=0.71075 \AA)$ 
graphite monochromated

Take-off Angle

$2.8^{\circ}$

Detector Aperture

2.0-2.5 $\mathrm{mm}$ horizontal

$2.0 \mathrm{~mm}$ vertical

Crystal to Detector Distance

$21 \mathrm{~mm}$

Temperature

$24.9{ }^{\circ} \mathrm{C}$

Scan Type

$\mathrm{w}-2 \mathrm{q}$

$2 \theta_{\max }$

$55.0^{\circ}$

Corrections

Lorentz-polarization

Absorption

(trans. factors: 0.860-0.940)

\section{Structure Solution and Refinement}

Structure Solution

Refinement

Function Minimized

Least Squares Weights

$2 \theta_{\max }$ cutoff

Anomalous Dispersion

No. Observations (I>2.00 $\sigma(\mathrm{I}))$

No. Variables

Reflection/Parameter Ratio

Residuals: R (I>2.00 $\sigma(\mathrm{I}))$

Residuals: $\mathrm{R}_{\mathrm{w}}(\mathrm{I}>2.00 \sigma(\mathrm{I}))$

Goodness of Fit Indicator

Max Shift/Error in Final Cycle

Maximum peak in Final Diff. Map

Minimum peak in Final Diff. Map
Direct Methods (SIR92)

Full-matrix least-squares on $\mathrm{F}$

$\Sigma \omega\left(\left|\mathrm{F}_{\mathrm{o}}\right|-\left|\mathrm{F}_{\mathrm{c}}\right|\right)^{2}$

1

$55.0^{\circ}$

All non-hydrogen atoms

3946

277

14.25

0.0502

0.1105

1.047

0.000

$0.20 \mathrm{e}^{-} / \AA^{3}$

$-0.20 \mathrm{e}^{-} / \AA^{3}$ 


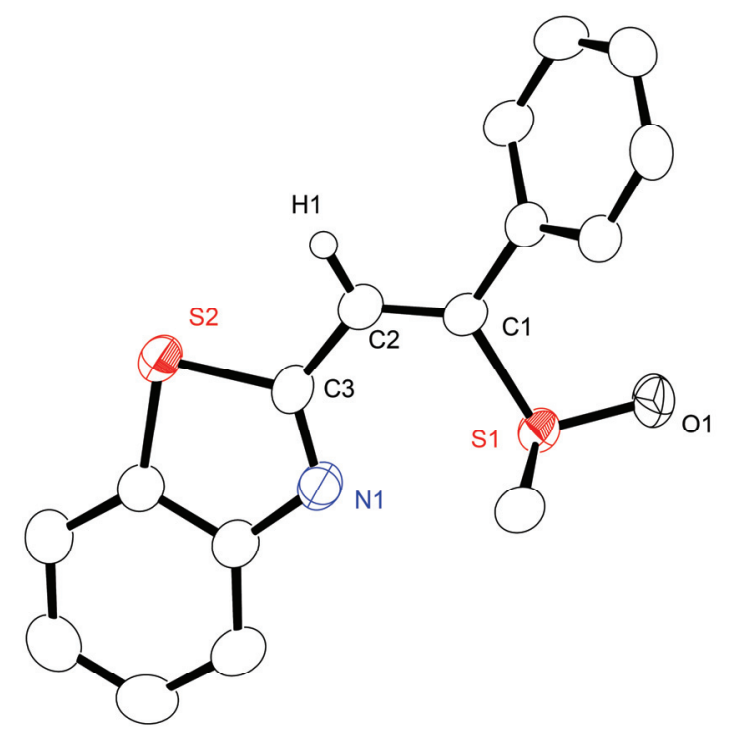

Figure S1. An ORTEP Drawing of 6.

\section{A. Crystal Data}

Empirical Formula

$\mathrm{C}_{16} \mathrm{H}_{13} \mathrm{NOS}_{2}$

Formula Weight

299.40

Crystal Color, Habit

yellow, prism

Crystal Dimensions

$0.50 \times 0.40 \times 0.15 \mathrm{~mm}$

Crystal System

monoclinic

Lattice Type

Primitive

No. of Reflections Used for Unit

Cell Determination ( $2 \theta$ range)

$10855\left(6.1-55.0^{\circ}\right)$

Lattice Parameters

$\mathrm{a}=11.330(5) \AA$

$\mathrm{b}=7.224(3) \AA$

$\mathrm{c}=17.293(10) \AA$

$\mathrm{V}=1400.6(12) \AA^{3}$

Space Group

$P 2{ }_{1} / \mathrm{c}(\# 14)$

$Z$ value

4

$\mathrm{D}_{\text {calc }}$

$1.420 \mathrm{~g} / \mathrm{cm}^{3}$

$F_{000}$

624.00

$\mu(\operatorname{MoK} \alpha)$

$3.734 \mathrm{~cm}^{-1}$

\section{B. Intensity Measurements}

Diffractometer

AFC7

Radiation

$\operatorname{MoK} \alpha(\lambda=0.71075 \AA)$ 
graphite monochromated

Take-off Angle

$2.8^{\circ}$

Detector Aperture

2.0-2.5 $\mathrm{mm}$ horizontal

$2.0 \mathrm{~mm}$ vertical

Crystal to Detector Distance

$21 \mathrm{~mm}$

Temperature

$24.9{ }^{\circ} \mathrm{C}$

Scan Type

$\mathrm{w}-2 \theta$

$2 \theta_{\max }$

$55.0^{\circ}$

Corrections

Lorentz-polarization

Absorption

(trans. factors: 0.835-0.946)

\section{Structure Solution and Refinement}

Structure Solution

Refinement

Function Minimized

Least Squares Weights

$2 \theta_{\max }$ cutoff

Anomalous Dispersion

No. Observations (I>2.00 $\sigma(\mathrm{I}))$

No. Variables

Reflection/Parameter Ratio

Residuals: R (I>2.00 $\sigma(\mathrm{I}))$

Residuals: $\mathrm{R}_{\mathrm{w}}(\mathrm{I}>2.00 \sigma(\mathrm{I}))$

Goodness of Fit Indicator

Max Shift/Error in Final Cycle

Maximum peak in Final Diff. Map

Minimum peak in Final Diff. Map
Direct Methods (SIR92)

Full-matrix least-squares on $\mathrm{F}$

$\Sigma \omega(|\mathrm{Fo}|-|\mathrm{Fc}|)^{2}$

1

$55.0^{\circ}$

All non-hydrogen atoms

3170

233

13.61

0.0340

0.0946

1.050

0.001

$0.35 \mathrm{e}^{-} / \AA^{3}$

$-0.26 \mathrm{e}^{-} / \AA^{3}$ 


\section{References}

1. Nasielski, J.; Hadei, N.; Achonduh, G.; Kantchev, E. A. B.; O’Brien, C. J.; Lough, A.; Organ, M. G. Chem. Eur. J. 2010, 16, 10844-10853.

2. Časar, Z.; Lorcy, D.; Leban, I.; Maréchal, A. M.-L. Acta Chim. Slov. 2002, 49, 871-883.

3. Popov, I.; Do, H.-Q.; Daugulis, O. J. Org. Chem. 2009, 74, 8309-8313.

4. Arisawa, M.; Toriyama, F.; Yamaguchi, M. Tetrahedron Lett. 2011, 52, 2344-2347.

5. Blackman, A. J.; Bowie, J. H. Austr. J. Chem. 1972, 25, 335-343.

6. Dou H. J. M.; Hassanaly, P; Kister, J; Verrnin, G; Metzger, J. Helv. Chim. Acta. 1978, 61, 31433148 .

7. Brandsma, L.; De Jong, R. L. P.; VerKruijsse, H. D. Synthesis 1985, 948-949.

8. Kamat, M. N.; Rath, N. P.; Demchenko, A. V. J. Org. Chem. 2007, 72, 6938-6946.

9. Capon, B.; Lew, S. Q. Tetrahedron 1992, 48, 7823-7838.

10. Ookubo, Y.; Wakamiya, A.; Yorimitsu, H.; Osuka, A. Chem. Eur. J. 2012, 18, 12690-12697.

11. Kundu, H. G.; Nandi, B. J. Org. Chem. 2001, 66, 4563-4575.

12. Iwasaki, M.; Fujino, D.; Wada, T.; Kondoh, A.; Yorimitsu, H.; Oshima, K. Chem. Asian J. 2011, 6, 3190-3194.

13. For selected reviews on the Pummerer reaction, see: (a) Bur, S. K.; Padwa, A. Chem. Rev. 2004, 104, 2401-2432. (b) Feldman, K. S. Tetrahedron 2006, 62, 5003-5034. (c) Akai, S.; Kita, Y. Top. Curr. Chem. 2007, 274, 35-76. (d) Smith, L. H. S.; Coote, S. C.; Sneddon, H. F.; Procter, D. J. Angew. Chem. Int. Ed. 2010, 49, 5832-5844.

14. (a) Yoshida, S.; Yorimitsu, H.; Oshima, K. Org. Lett. 2009, 11, 2185-2188. (b) Eberhart, A. J.; Imbriglio, J. E.; Procter, D. J. Org. Lett. 2011, 13, 5882-5885. (c) Eberhart, A. J.; Cicoira, C.; Procter, D. J. Org. Lett. 2013, 15, 3994-3997.

15. (a) Tomita, K.; Terada, A.; Tachikawa, R. Heterocycles 1976, 4, 733-737. (b) Bycroft, B. W.; Landon, W. J. Chem. Soc., Chem. Commun. 1970, 967-968. (c) Harvey, J. N.; Viehe, H. G. J. Chem. Soc., Chem. Commun. 1995, 2345-2346. 